TRANSACTIONS OF THE

AMERICAN MATHEMATICAL SOCIETY

Volume 365, Number 1, January 2013, Pages 511-530

S 0002-9947(2012)05642-X

Article electronically published on July 25, 2012

\title{
COMPLEX SYMMETRIC WEIGHTED SHIFTS
}

\author{
SEN ZHU AND CHUN GUANG LI
}

\begin{abstract}
An operator $T$ on a complex Hilbert space $\mathcal{H}$ is said to be complex symmetric if there exists a conjugate-linear, isometric involution $C: \mathcal{H} \longrightarrow \mathcal{H}$ so that $C T C=T^{*}$. In this paper, it is completely determined when a scalar (unilateral or bilateral) weighted shift is complex symmetric. In particular, we give a canonical decomposition of weighted shifts with complex symmetry. Also we characterize those weighted shifts for which complex symmetry is invariant under generalized Aluthge transforms. As an application, we give a negative answer to a question of S. Garcia.
\end{abstract}

\section{INTRODUCTION}

In their paper [7, Garcia and Putinar initiated the study for complex symmetric operators on complex Hilbert spaces which have many motivations in function theory, matrix analysis and other areas. Some important results concerning the internal structure of complex symmetric operators have been obtained (see [7, 8, 12, 9, 5, 10, 4, 11, 3, for references). To proceed, we first introduce some notation and terminology.

Throughout this paper, we let $\mathbb{C}, \mathbb{N}$ and $\mathbb{Z}$ denote the set of complex numbers, the set of positive integers and the set of integers respectively. We always denote by $\mathcal{H}$ a complex separable Hilbert space, and by $\mathcal{B}(\mathcal{H})$ the algebra of all bounded linear operators on $\mathcal{H}$. In what follows, the word operator will always mean a bounded linear operator.

Definition 1.1. A conjugation on $\mathcal{H}$ is a conjugate-linear map $C: \mathcal{H} \longrightarrow \mathcal{H}$ satisfying that $C^{2}=I$ and $(C x, C y)=(y, x)$ for all $x, y \in \mathcal{H}$.

Definition 1.2. We say that an operator $T \in \mathcal{B}(\mathcal{H})$ is complex symmetric, denoted by $T \in(\mathrm{CS})$, if there exists a conjugation $C$ on $\mathcal{H}$ so that $C T C=T^{*}$. In this case, $T$ is said to be $C$-symmetric.

Garcia and Putinar [7] indicated that an operator $T$ on $\mathcal{H}$ is complex symmetric if and only if there exists an orthonormal basis (ONB for short) $\left\{e_{n}\right\}$ of $\mathcal{H}$ such that $\left(T e_{i}, e_{j}\right)=\left(T e_{j}, e_{i}\right)$ for all $i, j$, that is, $T$ admits a symmetric matrix representation with respect to $\left\{e_{n}\right\}$. Although much attention has been paid to complex symmetric operators, the internal structure of complex symmetric operators is still not well

Received by the editors April 8, 2011 and, in revised form, May 27, 2011.

2010 Mathematics Subject Classification. Primary 47B37, 47A05; Secondary 47A66.

Key words and phrases. Complex symmetric operator, weighted shift, nilpotent operator, Aluthge transform, generalized Aluthge transform.

This work was partially supported by NNSF of China (11101177, 11026038, 10971079), China Postdoctoral Science Foundation (2011M500064) and Shanghai Postdoctoral Scientific Program (12R21410500). 
understood. In particular, Garcia [6] posed many concrete questions concerning complex symmetric operators.

An effective way to investigate the structure of complex symmetric operators is to characterize which special operators are complex symmetric. Through a series of papers, many important operators, such as Hankel operators, truncated Toeplitz operators, normal operators and binormal operators, have proved to be complex symmetric.

This paper is partially inspired by a recent paper of Garcia and Wogen 10, in which they gave a characterization of partial isometries being complex symmetric. The main aim of this paper is to give a characterization of weighted shifts being complex symmetric. Perhaps our results will provide many nontrivial examples for the further study of complex symmetric operators.

Recall that a (forward) weighted shift $T$ on $\mathcal{H}\left(\operatorname{dim} \mathcal{H}=\aleph_{0}\right)$ with weighted sequence $\left\{w_{n}\right\}$ is the operator defined by $T e_{n}=w_{n} e_{n+1}$ for all $n$, where $\left\{e_{n}\right\}$ is an ONB of $\mathcal{H}$. If the index $n$ runs over the positive integers, then $T$ is called a unilateral weighted shift, while if $n$ runs over integers, then $T$ is called a bilateral weighted shift. According to a result of Shields [16, Corollary 1], the above weighted shift is unitarily equivalent to the weighted shift defined as $A e_{n}=\left|w_{n}\right| e_{n+1}$ for all $n$. On the other hand, it is obvious that complex symmetry is invariant under unitary equivalence. Thus we may often deal with weighted shifts whose weights are nonnegative.

Note that if $T$ is a unilateral weighted shift, then $T$ is injective if and only if all weights are nonzero if and only if $T$ is irreducible. In most studies of weighted shifts, one considers only the irreducible ones. As we shall see in Section 3, the nontrivial cases in this study involve shifts with many weights being zero. Thus for the most part we study when direct sums of finite-dimensional truncated weighted shifts (as described at the beginning of Section 2) are complex symmetric.

Let $T \in \mathcal{B}(\mathcal{H})$. Assume that $T=U|T|$ is the polar decomposition of $T$. If $\varepsilon \in[0,1]$, then the operator $T_{\varepsilon}=|T|^{\varepsilon} U|T|^{1-\varepsilon}$ is called the generalized Aluthge transform of $T$ of order $\varepsilon$. In particular, $|T|^{\frac{1}{2}} U|T|^{\frac{1}{2}}$ is called the Aluthge transform of $T$.

The Aluthge transform and its generalizations originally arose in the study of $p$ hyponormal operators [1, 2]. In 2008, Garcia [5] proved that the Aluthge transform of a complex symmetric operator is always complex symmetric. Also he presented an open question.

Question 1.3. If $T \in \mathcal{B}(\mathcal{H})$ is complex symmetric and $0<\varepsilon<1 / 2$, is it necessarily the case that $|T|^{\varepsilon} U|T|^{1-\varepsilon}$ is also complex symmetric?

In 2009, Wang and Gao [17 proved that if $T \in \mathcal{B}(\mathcal{H})$ is complex symmetric and $T=U|T|$ is the polar decomposition of $T$, then $|T|^{\varepsilon} U|T|^{\varepsilon}$ is complex symmetric for all $\varepsilon \in[0,1]$.

In this paper, we shall also characterize those weighted shifts $T$ satisfying that $T_{\varepsilon} \in(\mathrm{CS})$ for all $\varepsilon \in[0,1]$. As an application, we give a negative answer to Question 1.3 (see Example 5.4).

The rest of this paper is organized as follows. In Section 2, we shall make some preparations. Section 3 is devoted to the characterization of unilateral weighted shifts being complex symmetric (Theorem 3.1). In Section 4, we shall characterize complex symmetric bilateral weighted shifts (Theorem 4.1). In the last section, we shall study the generalized Aluthge transforms of weighted shifts. 


\section{Preparations}

Given a set $E$, we denote by card $E$ the cardinality of $E$. Given an operator $T$, we denote by $\operatorname{ker} T$ and $\operatorname{ran} T$ the kernel of $T$ and the range of $T$ respectively. Also we denote $\operatorname{nul} T=\operatorname{dim} \operatorname{ker} T$. Given a nonempty subset $M$ of $\mathcal{H}$, we denote by $\vee M$ the closed linear span of all vectors in $M$.

Given $e, f \in \mathcal{H}$, we let $e \otimes f$ denote the finite-rank operator on $\mathcal{H}$ defined as $(e \otimes f)(x)=(x, f) e, \forall x \in \mathcal{H}$. Let $n \in \mathbb{N}$ and $\left\{e_{i}\right\}_{i=1}^{n}$ be an ONB of $\mathbb{C}^{n}$. If $n \geq 2$, then the operator $T=\sum_{i=1}^{n-1} \lambda_{i} e_{i} \otimes e_{i+1}$ admits the following matrix representation:

$$
T=\left[\begin{array}{cccc}
0 & \lambda_{1} & & \\
& \ddots & \ddots & \\
& & 0 & \lambda_{n-1} \\
& & & 0
\end{array}\right] \begin{gathered}
e_{1} \\
\vdots \\
e_{n-1} \\
e_{n}
\end{gathered} .
$$

Here $\left\{\lambda_{i}\right\}_{i=1}^{n-1} \subset \mathbb{C}$. When $n=1$, we also write the above matrix to denote the 0 operator on the one-dimensional Hilbert space $\mathbb{C}$. We note that the above operator is a finite-dimensional truncated weighted shift (see [13, 14]).

For convenience, we list some useful results.

Lemma 2.1 (] 8 , Theorem 2). Let $T \in \mathcal{B}(\mathcal{H})$. If $T=U|T|$ is the polar decomposition of a $C$-symmetric operator $T$, then $C U$ commutes with $|T|$.

Lemma 2.2 ([1], Theorem 2). If $T \in \mathcal{B}(\mathcal{H})$ and $T^{2}=0$, then $T \in(\mathrm{CS})$.

Lemma 2.3 ([7], Proposition 1). Let $T \in \mathcal{B}(\mathcal{H})$ and $C$ be a conjugation on $\mathcal{H}$. If $T$ is $C$-symmetric, then $C\left[\operatorname{ker}(T-\lambda)^{k}\right]=\operatorname{ker}\left(T^{*}-\bar{\lambda}\right)^{k}$ for all $k \geq 1$ and $\lambda \in \mathbb{C}$. In particular, $T \in(\mathrm{CS})$ implies that $\operatorname{nul}(T-\lambda)^{k}=\operatorname{nul}\left(T^{*}-\bar{\lambda}\right)^{k}$ for all $k \geq 1$ and $\lambda \in \mathbb{C}$.

Lemma 2.4. Let $T=\bigoplus_{i \in \Gamma} T_{i}$, where $1 \leq \operatorname{card} \Gamma \leq \aleph_{0}$. If $T_{i} \in(\mathrm{CS})$ for all $i \in \Gamma$, then $T \in(\mathrm{CS})$.

Proof. For $i \in \Gamma$, denote by $\mathcal{H}_{i}$ the underlying space of $T_{i}$ and assume that $C_{i}$ is a conjugation on $\mathcal{H}_{i}$ such that $C_{i} T_{i} C_{i}=T_{i}^{*}$. Then it is obvious that $C:=\bigoplus_{i \in \Gamma} C_{i}$ is a conjugation on $\bigoplus_{i \in \Gamma} \mathcal{H}_{i}$ satisfying $C T C=T^{*}$.

Lemma 2.5 ([10, Lemma 1$)$. Let $T \in \mathcal{B}(\mathcal{H})$. If $M$ is a reducing subspace of $T$ and $\left.T\right|_{M}=0$, then $T \in(\mathrm{CS})$ if and only if $\left.T\right|_{M^{\perp}} \in(\mathrm{CS})$.

It is easy to check that if $T \in \mathcal{B}(\mathcal{H})$, then $T$ can be written as $T=T_{1} \oplus$ $T_{2}$, where $T_{1}$ is normal and $T_{2}$ has no nontrivial reducing subspace $M$ such that $\left.T_{2}\right|_{M}$ is normal. $T_{1}$ and $T_{2}$ are called the normal part and the abnormal part of $T$ respectively (see [15, page 116]). (Of course, $T_{1}$ or $T_{2}$ can be absent.) Note that the normal part of a unilateral weighted shift (if it exists) is always 0 . Then the following corollary is clear.

Corollary 2.6. $\quad$ (i) Let $T$ be a unilateral weighted shift. Then $T$ is complex symmetric if and only if the abnormal part of $T$ is complex symmetric.

(ii) Let $n \in \mathbb{N}, n \geq 2$ and $\left\{e_{i}\right\}_{i=1}^{n}$ be an ONB of $\mathbb{C}^{n}$. Assume that $\left\{w_{i}\right\}_{i=1}^{n-1} \subset \mathbb{C}$ and $A=\sum_{i=1}^{n-1} w_{i} e_{i} \otimes e_{i+1}$. Then $A$ is complex symmetric if and only if the abnormal part of $A$ is complex symmetric. 
Let $n \in \mathbb{N}$ and $T$ be a nilpotent operator of order $n$ on $\mathcal{H}$, that is, $T^{n}=0$ and $T^{n-1} \neq 0$. If $n=1$, then $T=0$; if $n \geq 2$, then it is obvious that $T$ admits the following matrix representation:

$$
T=\left[\begin{array}{ccccc}
0 & A_{1} & * & \cdots & * \\
& 0 & A_{2} & \cdots & * \\
& & \ddots & \ddots & \vdots \\
& & & 0 & A_{n-1} \\
& & & & 0
\end{array}\right] \begin{gathered}
\mathcal{H}_{1} \\
\mathcal{H}_{2} \\
\vdots \\
\mathcal{H}_{n-1}
\end{gathered}
$$

where $\mathcal{H}_{i}=\operatorname{ker} T^{i} \ominus \operatorname{ker} T^{i-1}$ for $i=1,2, \ldots, n$. Then $A_{i}$ is a bounded linear operator mapping $\mathcal{H}_{i+1}$ into $\mathcal{H}_{i}$ and nul $A_{i}=0$ for $1 \leq i \leq n-1$. In what follows, we say that $T$ is a regular nilpotent operator if (i) $n=1$, or (ii) $\operatorname{ran} A_{i}$ is dense in $\mathcal{H}_{i}$ for $1 \leq i \leq n-1$. Then it can be seen that a nilpotent operator is regular if and only if its adjoint is regular.

Let $T$ be as above. Assume that $n \geq 2$ and $T$ is regular of order $n$. Then it is easy to verify that $\operatorname{ker} T^{i}=\bigoplus_{j=1}^{i} \mathcal{H}_{j}$ and $\operatorname{ker}\left(T^{*}\right)^{i}=\bigoplus_{j=n-i+1}^{n} \mathcal{H}_{j}$ for all $1 \leq i \leq n$; in particular, $\operatorname{ker} T^{i} \perp \operatorname{ker} T^{*}$ and $\operatorname{ker}\left(T^{*}\right)^{i} \perp \operatorname{ker} T$ for all $1 \leq i \leq n-1$.

Proposition 2.7. Let $T \in \mathcal{B}(\mathcal{H})$ have the form of $T=\bigoplus_{1 \leq i<\omega} T_{i}$, where $\omega \in \mathbb{N}$ or $\omega=\infty, T_{i}$ is a regular nilpotent operator of order $n_{i}$ and $n_{i} \neq n_{j}$ for $1 \leq i \neq j<\omega$. Then $T \in(\mathrm{CS})$ if and only if $T_{i} \in(\mathrm{CS})$ for all $1 \leq i<\omega$.

Proof. The sufficiency is obvious. We need only prove the necessity.

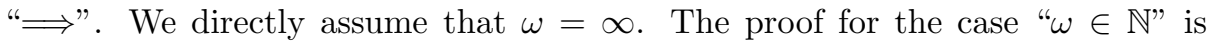
similar. Since $T \in(\mathrm{CS})$, there exists a conjugation $C$ on $\mathcal{H}$ such that $C T=T^{*} C$. For each $i \in \mathbb{N}$, denote by $\mathcal{H}_{i}$ the underlying space of $T_{i}$. Without loss of generality, we assume that $n_{i}<n_{i+1}$ for all $i \in \mathbb{N}$. By Lemma 2.5, we may also assume that $n_{i} \geq 2$ for all $i$.

One can note that it suffices to prove that $C\left(\mathcal{H}_{i}\right)=\mathcal{H}_{i}$ for all $i \in \mathbb{N}$. In fact, if this holds, then it is easy to verify that the map $C_{i}:=\left.C\right|_{\mathcal{H}_{i}}$ is a conjugation on $\mathcal{H}_{i}$ for each $i \in \mathbb{N}$. Hence, for each $i$ and $x \in \mathcal{H}_{i}, C_{i} T_{i} x=C T x=T^{*} C x=T_{i}^{*} C_{i} x$. Then $T_{i}$ is complex symmetric for all $i \in \mathbb{N}$.

We shall prove the above statement step by step.

Step 1. $C\left(\operatorname{ker} T_{i}\right)=\operatorname{ker} T_{i}^{*}$ for all $i \in \mathbb{N}$.

Let $i \in \mathbb{N}$ be fixed. Arbitrarily choose an $x \in \operatorname{ker} T_{i}$. Note that $\operatorname{ker} T_{i} \subset \operatorname{ker} T$. Then $T^{*} C x=C T x=C T_{i} x=0$ and $C x \in \operatorname{ker} T^{*}=\bigoplus_{j \geq 1} \operatorname{ker} T_{j}^{*}$. Assume that $C x=\sum_{j \geq 1} x_{j}$, where $x_{j} \in \operatorname{ker} T_{j}^{*}$ for all $j \in \mathbb{N}$. Note that $T_{j}^{n_{i}}=0$ for all $1 \leq j \leq i$. Then

$$
\begin{aligned}
0=C\left(T_{i}^{n_{i}}\right)^{*} x & =C\left(T^{n_{i}}\right)^{*} x=T^{n_{i}} C x \\
& =\sum_{j \geq 1} T^{n_{i}} x_{j}=\sum_{j \geq 1} T_{j}^{n_{i}} x_{j}=\sum_{j \geq i+1} T_{j}^{n_{i}} x_{j} .
\end{aligned}
$$

Since $T_{j}^{n_{i}} x_{j} \in \mathcal{H}_{j}$ for $j \in \mathbb{N}$ and all $\mathcal{H}_{j}$ are pairwise orthogonal, we deduce that $T_{j}^{n_{i}} x_{j}=0$ for all $j \geq i+1$. Note that $T_{j}$ is of order $n_{j}$ and $n_{j}>n_{i}$ for $j \geq i+1$. Then $\operatorname{ker} T_{j}^{n_{i}} \perp \operatorname{ker} T_{j}^{*}$ for $j \geq i+1$. This implies that $x_{j}=0$ for all $j \geq i+1$ and $C x=\sum_{j=1}^{i} x_{j} \in \bigoplus_{j=1}^{i} \operatorname{ker} T_{j}^{*}$. Thus we have proved that $C\left(\operatorname{ker} T_{i}\right) \subset \bigoplus_{j=1}^{i} \operatorname{ker} T_{j}^{*}$.

Then $C\left(\operatorname{ker} T_{1}\right) \subset \operatorname{ker} T_{1}^{*}$. Applying the same argument to $T^{*}$, one can obtain $C\left(\operatorname{ker} T_{1}^{*}\right) \subset \operatorname{ker} T_{1}$. Note that $C^{2}=I$, and we have $C\left(\operatorname{ker} T_{1}\right)=\operatorname{ker} T_{1}^{*}$. Since 
$C\left(\operatorname{ker} T_{2}\right) \subset \operatorname{ker} T_{1}^{*} \oplus \operatorname{ker} T_{2}^{*}$, we have $C\left(\operatorname{ker} T_{2}\right) \subset \operatorname{ker} T_{2}^{*}$. By the symmetry of $T_{2}$ and $T_{2}^{*}$, we have $C\left(\operatorname{ker} T_{2}\right)=\operatorname{ker} T_{2}^{*}$. An induction $\operatorname{argument}$ shows that $C\left(\operatorname{ker} T_{i}\right)=$ $\operatorname{ker} T_{i}^{*}$ for all $i \geq 1$.

Step 2. Assume that $n \in \mathbb{N}$ and $C\left(\operatorname{ker} T_{i}^{k}\right)=\operatorname{ker}\left(T_{i}^{k}\right)^{*}$ for all $i \in \mathbb{N}, k \leq n$. We shall prove that $C\left(\operatorname{ker} T_{i}^{n+1}\right)=\operatorname{ker}\left(T_{i}^{n+1}\right)^{*}$ for all $i \in \mathbb{N}$.

Let $i \in \mathbb{N}$ be fixed. Arbitrarily choose an $x \in \operatorname{ker} T_{i}^{n+1} \ominus \operatorname{ker} T_{i}^{n}$. Then $T^{n+1} x=$ $T_{i}^{n+1} x=0$ and hence $C x \in \operatorname{ker}\left(T^{n+1}\right)^{*}=\bigoplus_{j \geq 1} \operatorname{ker}\left(T_{j}^{n+1}\right)^{*}$. Assume that $C x=$ $\sum_{j \geq 1} x_{j}$, where $x_{j} \in \operatorname{ker}\left(T_{j}^{n+1}\right)^{*}$ for all $j \in \mathbb{N}$. Then $C T_{i} x=C T x=T^{*} C x=$ $\sum_{j \geq 1} T^{*} x_{j}=\sum_{j \geq 1} T_{j}^{*} x_{j}$.

Note that $T_{i} x \in \operatorname{ker} T_{i}^{n}$. Then, by the induction hypothesis, we have $C T_{i} x \in$ $\operatorname{ker}\left(T_{i}^{n}\right)^{*} \subset \mathcal{H}_{i}$. Thus we deduce that $T_{j}^{*} x_{j}=0$ and $x_{j} \in \operatorname{ker} T_{j}^{*}$ for all $j \neq i$. Since $x \in \operatorname{ker} T_{i}^{n+1} \ominus \operatorname{ker} T_{i}^{n}$, we have $x \in(\operatorname{ker} T)^{\perp}$ and hence $C x \in[C(\operatorname{ker} T)]^{\perp}=$ $\left(\operatorname{ker} T^{*}\right)^{\perp}=\left[\bigoplus_{j \geq 1} \operatorname{ker} T_{j}^{*}\right]^{\perp}$. Thus we have $\left\|x_{j}\right\|^{2}=\left(C x, x_{j}\right)=0$ for $j \neq i$. Then $C x=x_{i} \in \operatorname{ker}\left(T_{i}^{n+1}\right)^{*}$. Hence $C\left(\operatorname{ker} T_{i}^{n+1} \ominus \operatorname{ker} T_{i}^{n}\right) \subset \operatorname{ker}\left(T_{i}^{n+1}\right)^{*}$ and $C\left(\operatorname{ker} T_{i}^{n+1}\right) \subset \operatorname{ker}\left(T_{i}^{n+1}\right)^{*}$.

Applying the above $\operatorname{argument}$ to $T^{*}$, we can prove that $C\left[\operatorname{ker}\left(T_{i}^{n+1}\right)^{*}\right] \subset \operatorname{ker} T_{i}^{n+1}$, and hence $C\left(\operatorname{ker} T_{i}^{n+1}\right)=\operatorname{ker}\left(T_{i}^{n+1}\right)^{*}$.

Using an induction argument, we deduce that $C\left(\operatorname{ker} T_{i}^{n+1}\right)=\operatorname{ker}\left(T_{i}^{n+1}\right)^{*}$ for all $n \in \mathbb{N}$ and all $i \in \mathbb{N}$. Note that $\operatorname{ker} T_{i}^{n_{i}}=\mathcal{H}_{i}=\operatorname{ker}\left(T_{i}^{*}\right)^{n_{i}}$ for $i \in \mathbb{N}$. Then we have proved that $C\left(\mathcal{H}_{i}\right)=\mathcal{H}_{i}$ for all $i \in \mathbb{N}$. This completes the proof.

The following result is essentially contained in a recent paper of the authors and Ji [18. For completeness, we still present it here.

Lemma 2.8. Let $n \in \mathbb{N}$ and $\left\{e_{i}\right\}_{i=1}^{n}$ be an othonormal basis (ONB for short) of $\mathbb{C}^{n}$. Assume that $T \in \mathcal{B}\left(\mathbb{C}^{n}\right)$ admits the following matrix representation:

$$
T=\left[\begin{array}{ccccc}
0 & \lambda_{1} & & & \\
& 0 & \lambda_{2} & & \\
& & \ddots & \ddots & \\
& & & 0 & \lambda_{n-1} \\
& & & & 0
\end{array}\right] \begin{gathered}
e_{1} \\
e_{2} \\
\vdots \\
e_{n-1} \\
e_{n}
\end{gathered},
$$

where $\left|\lambda_{i}\right|=\left|\lambda_{n-i}\right|$ for all $1 \leq i \leq n-1$. Then $T$ is complex symmetric.

Proof. Without loss of generality, we may directly assume that $n \geq 2$ and $\lambda_{i}=$ $\left|\lambda_{i}\right|$ for all $1 \leq i \leq n-1$. In fact, $T$ is unitarily equivalent to the operator $\sum_{i=1}^{n-1}\left|\lambda_{i}\right| e_{i} \otimes e_{i+1}$.

For $x \in \mathbb{C}^{n}, x=\sum_{i=1}^{n} \alpha_{i} e_{i}$, define $C x=\sum_{i=1}^{n} \overline{\alpha_{i}} e_{n-i+1}$. Obviously, $C$ is a conjugation on $\mathbb{C}^{n}$ and $C e_{i}=e_{n-i+1}$ for $1 \leq i \leq n$. It suffices to verify that $C T C=T^{*}$. Since $C T C$ and $T^{*}$ are both linear on $\mathbb{C}^{n}$, we need only prove that $C T C e_{i}=T^{*} e_{i}$ for all $i$.

First, one can note that $T^{*}=\sum_{i=1}^{n-1} \lambda_{i} e_{i+1} \otimes e_{i}$. Then $C T C e_{n}=C T e_{1}=0=$ $T^{*} e_{n}$.

Let $1 \leq i \leq n-1$ be fixed. Then, by the definition of $T$,

$$
C T C e_{i}=C T e_{n-i+1}=C\left(\lambda_{n-i} e_{n-i}\right)=\lambda_{n-i} e_{i+1},
$$

and, by the definition of $T^{*}, T^{*} e_{i}=\lambda_{i} e_{i+1}$. Note that $\lambda_{i}=\lambda_{n-i}$; thus we have $C T C e_{i}=T^{*} e_{i}$. This completes the proof. 
Remark 2.9. As we shall see in the latter, any complex symmetric unilateral weighted shift can be represented as the orthogonal direct sum of complex symmetric operators with the form of the operator in Lemma 2.8

\section{UNilateral CASE}

The main result of this section is the following theorem, which gives a canonical decomposition of complex symmetric unilateral weighted shifts.

Theorem 3.1. Let $T$ be a unilateral weighted shift on $\mathcal{H}$, where $\operatorname{dim} \mathcal{H}=\aleph_{0}$. Then $T$ is complex symmetric if and only if $T$ can be written as $T=\bigoplus_{i=1}^{\infty} T_{i}$, where each $T_{i}$ is acting on a finite-dimensional Hilbert space and admits the following matrix representation:

$$
T_{i}=\left[\begin{array}{ccccc}
0 & \lambda_{1}^{(i)} & & & \\
& 0 & \lambda_{2}^{(i)} & & \\
& & \ddots & \ddots & \\
& & & 0 & \lambda_{n_{i}-1}^{(i)} \\
& & & & 0
\end{array}\right]
$$

with respect to some ONB of the underlying space of $T_{i}$ and $\left|\lambda_{j}^{(i)}\right|=\left|\lambda_{n_{i}-j}^{(i)}\right|$ for all $1 \leq j \leq n_{i}-1$.

For $n \in \mathbb{N}$ and an $n$-tuple $\mathbf{a}=\left(a_{1}, a_{2}, \cdots, a_{n}\right)$ of complex numbers, we denote $\mathbf{a}^{t}=\left(b_{1}, b_{2}, \cdots, b_{n}\right)$, where $b_{i}=a_{n-i+1}$ for $1 \leq i \leq n$.

Proposition 3.2. Let $n \in \mathbb{N}$. Assume that $T=\bigoplus_{i \in \Gamma} T_{i}$, where $1 \leq \operatorname{card} \Gamma \leq \aleph_{0}$,

$$
T_{i}=\left[\begin{array}{cccc}
0 & \lambda_{1}^{(i)} & & \\
& \ddots & \ddots & \\
& & 0 & \lambda_{n}^{(i)} \\
& & & 0
\end{array}\right] \begin{gathered}
e_{1}^{i} \\
\vdots \\
e_{n+1}^{i} \\
e_{n+1}^{i}
\end{gathered}
$$

with respect to some ONB $\left\{e_{j}^{i}\right\}_{j=1}^{n+1}$ of the underlying space of $T_{i}$ and $\operatorname{nul} T_{i}=$ 1 for each $i \in \Gamma$. Then $T$ is complex symmetric if and only if $\operatorname{card}\{i \in \Gamma$ : $\left.\left(\left|\lambda_{1}^{(i)}\right|, \cdots,\left|\lambda_{n}^{(i)}\right|\right)=\mathbf{a}\right\}=\operatorname{card}\left\{i \in \Gamma:\left(\left|\lambda_{1}^{(i)}\right|, \cdots,\left|\lambda_{n}^{(i)}\right|\right)=\mathbf{a}^{t}\right\}$ for any $n$-tuple a of complex numbers.

Proof. In view of Lemma 2.2, the result is trivial in the case that $n=1$. We may directly assume that $n \geq 2$. On the other hand, we may also assume that $\lambda_{j}^{(i)}>0$ for all $i, j$. For each $i \in \Gamma$, denote $\mathbf{a}_{i}=\left(\lambda_{1}^{(i)}, \lambda_{2}^{(i)}, \cdots, \lambda_{n}^{(i)}\right)$.

"£". Denote $\Gamma_{1}=\left\{i \in \Gamma: \mathbf{a}_{i}=\mathbf{a}_{i}^{t}\right\}$ and $\Gamma_{2}=\Gamma \backslash \Gamma_{1}$. Then, by Lemma 2.8, $T_{i}$ is complex symmetric for all $i \in \Gamma_{1}$.

By hypothesis, $\operatorname{card}\left\{i \in \Gamma: \mathbf{a}_{i}=\mathbf{a}\right\}=\operatorname{card}\left\{i \in \Gamma: \mathbf{a}_{i}=\mathbf{a}^{t}\right\}$ for any $n$-tuple $\mathbf{a}$. If $i \in \Gamma_{2}$, then $\mathbf{a}_{i} \neq \mathbf{a}_{i}^{t}$ and hence there exists $k \in \Gamma_{2}$ such that $\mathbf{a}_{i}=\mathbf{a}_{k}^{t}$. Moreover, there exists a partition $\Delta=\left\{\Delta_{j}: 1 \leq j<\omega\right\}(\omega \in \mathbb{N}$ or $\omega=\infty)$ of $\Gamma_{2}$ such that (i) card $\Delta_{j}=2$ for all $j$, and (ii) if $i, k \in \Delta_{j}, i \neq k$, for some $j$, then $\mathbf{a}_{i}=\mathbf{a}_{k}^{t}$.

By Lemma 2.8, $\bigoplus_{i \in \Delta_{j}} T_{i}$ is complex symmetric for all $1 \leq j<\omega$. Then

$$
\bigoplus_{i \in \Gamma_{2}} T_{i}=\bigoplus_{1 \leq j<\omega}\left(\bigoplus_{i \in \Delta_{j}} T_{i}\right)
$$


is complex symmetric. Furthermore, $T=\left(\bigoplus_{i \in \Gamma_{1}} T_{i}\right) \oplus\left(\bigoplus_{i \in \Gamma_{2}} T_{i}\right)$ is complex symmetric.

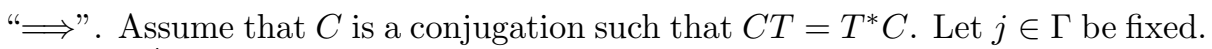
Obviously, $e_{1}^{j} \in \operatorname{ker} T$. Note that $\operatorname{ker} T^{*}=\bigvee\left\{e_{n+1}^{i}: i \in \Gamma\right\}$. Then, by Lemma 2.3. we may assume that $C e_{1}^{j}=\sum_{i \in \Gamma_{1}} \beta_{i} e_{n+1}^{i}$, where $\Gamma_{1}$ is a subset of $\Gamma$ and $\beta_{i} \neq 0$ for all $i \in \Gamma_{1}$.

For $1 \leq k \leq n$, it is easy to see that $C T^{k}=\left(T^{k}\right)^{*} C$, which means that $T^{k}$ is $C$-symmetric. Assume that $T^{k}=U_{k}\left|T^{k}\right|$ is the polar decomposition of $T^{k}$. Then a simple calculation shows that

$$
\left|T^{k}\right|=\bigoplus_{i \in \Gamma}\left[\begin{array}{cccccc}
0 & & & & & \\
& \ddots & & & & \\
& & 0 & & & \\
& & \alpha_{1}^{(i)} & & \\
& & & \ddots & \\
& & & & \alpha_{n-k+1}^{(i)}
\end{array}\right] \begin{gathered}
e_{1}^{i} \\
\vdots \\
e_{k}^{i} \\
e_{k+1}^{i} \\
\vdots \\
e_{n+1}^{i}
\end{gathered}
$$

where $\alpha_{l}^{(i)}=\prod_{m=l}^{l+k-1} \lambda_{m}^{(i)}$ for $1 \leq l \leq n-k+1$ and $i \in \Gamma$. Also we have $U_{k}=U^{k}$, where

$$
U=\bigoplus_{i \in \Gamma}\left[\begin{array}{ccccc}
0 & 1 & & & \\
& 0 & 1 & & \\
& & \ddots & \ddots & \\
& & & 0 & 1 \\
& & & & 0
\end{array}\right] \begin{gathered}
e_{1}^{i} \\
e_{2}^{i} \\
\vdots \\
e_{n}^{i} \\
e_{n+1}^{i}
\end{gathered}
$$

By Lemma 2.1. we have $C U_{k}\left|T^{k}\right|=\left|T^{k}\right| C U_{k}$. A simple calculation shows that

$$
C U_{k}\left|T^{k}\right| e_{k+1}^{j}=C U_{k} \alpha_{1}^{(j)} e_{k+1}^{j}=C \alpha_{1}^{(j)} e_{1}^{j}=\alpha_{1}^{(j)} \sum_{i \in \Gamma_{1}} \beta_{i} e_{n+1}^{i}
$$

and

$$
\left|T^{k}\right| C U_{k} e_{k+1}^{j}=\left|T^{k}\right| C e_{1}^{j}=\left|T^{k}\right|\left(\sum_{i \in \Gamma_{1}} \beta_{i} e_{n+1}^{i}\right)=\sum_{i \in \Gamma_{1}} \beta_{i} \alpha_{n-k+1}^{(i)} e_{n+1}^{i} .
$$

Arbitrarily choose an $i_{0} \in \Gamma_{1}$. It follows that $\beta_{i_{0}} \neq 0$. From the equation $C U_{k}\left|T^{k}\right| e_{k+1}^{j}=\left|T^{k}\right| C U_{k} e_{k+1}^{j}$, we obtain $\alpha_{1}^{(j)}=\alpha_{n-k+1}^{\left(i_{0}\right)}$, which means that $\prod_{m=1}^{k} \lambda_{m}^{(j)}=\prod_{m=n-k+1}^{n} \lambda_{m}^{\left(i_{0}\right)}$.

Now we conclude that $\prod_{m=1}^{k} \lambda_{m}^{(j)}=\prod_{m=n-k+1}^{n} \lambda_{m}^{\left(i_{0}\right)}$ for all $1 \leq k \leq n$ and hence

$$
\lambda_{1}^{(j)}=\lambda_{n}^{\left(i_{0}\right)}, \lambda_{2}^{(j)}=\lambda_{n-1}^{\left(i_{0}\right)}, \cdots, \lambda_{n}^{(j)}=\lambda_{1}^{\left(i_{0}\right)} .
$$

For any $n$-tuple $\mathbf{a}$, we denote $\Gamma_{\mathbf{a}}=\left\{i \in \Gamma: \mathbf{a}_{i}=\mathbf{a}\right\}$. By the above argument, we can see that $\Gamma_{\mathbf{a}}=\emptyset$ if and only if $\Gamma_{\mathbf{a}^{t}}=\emptyset$. If $\Gamma_{\mathbf{a}} \neq \emptyset$, then, as we have proved in the above argument, $C\left(\bigvee\left\{e_{1}^{i}: i \in \Gamma_{\mathbf{a}}\right\}\right) \subset \bigvee\left\{e_{n+1}^{i}: i \in \Gamma_{\mathbf{a}^{t}}\right\}$. It is easy to see that

$$
\begin{aligned}
\operatorname{card} \Gamma_{\mathbf{a}} & =\operatorname{dim} \bigvee\left\{e_{1}^{i}: i \in \Gamma_{\mathbf{a}}\right\}=\operatorname{dim} C\left(\bigvee\left\{e_{1}^{i}: i \in \Gamma_{\mathbf{a}}\right\}\right) \\
& \leq \operatorname{dim}\left(\bigvee\left\{e_{n+1}^{i}: i \in \Gamma_{\mathbf{a}^{t}}\right\}\right)=\operatorname{card} \Gamma_{\mathbf{a}^{t}}
\end{aligned}
$$

By the symmetry of $\mathbf{a}^{t}$ and $\mathbf{a}$, we have card $\Gamma_{\mathbf{a}}=\operatorname{card} \Gamma_{\mathbf{a}^{t}}$. This completes the proof. 
Theorem 3.3. Assume that $T=\bigoplus_{i \in \Gamma} A_{i}$, where $1 \leq \operatorname{card} \Gamma \leq \aleph_{0}$,

$$
A_{i}=\left[\begin{array}{cccc}
0 & \lambda_{1}^{(i)} & & \\
& \ddots & \ddots & \\
& & 0 & \lambda_{n_{i}-1}^{(i)} \\
& & & 0
\end{array}\right] \begin{gathered}
e_{1}^{i} \\
\vdots \\
e_{n_{i}-1}^{i} \\
e_{n_{i}}^{i}
\end{gathered}
$$

with respect to some ONB $\left\{e_{j}^{i}\right\}_{j=1}^{n_{i}}$ of the underlying space of $A_{i}$ and nul $A_{i}=$ $1, n_{i} \geq 2$ for each $i \in \Gamma$. Then $T$ is complex symmetric if and only if $\operatorname{card}\{i \in$ $\left.\Gamma:\left(\left|\lambda_{1}^{(i)}\right|, \cdots,\left|\lambda_{n_{i}-1}^{(i)}\right|\right)=\mathbf{a}\right\}=\operatorname{card}\left\{i \in \Gamma:\left(\left|\lambda_{1}^{(i)}\right|, \cdots,\left|\lambda_{n_{i}-1}^{(i)}\right|\right)=\mathbf{a}^{t}\right\}$ for any $n \in \mathbb{N}$ and any $n$-tuple a of complex numbers.

Proof. Without loss of generality, we may directly assume that $\left|\lambda_{j}^{(i)}\right|=\lambda_{j}^{(i)}$ for all $i, j$.

The sufficiency can be seen from the proof for the sufficiency of Proposition 3.2 We need only prove the necessity.

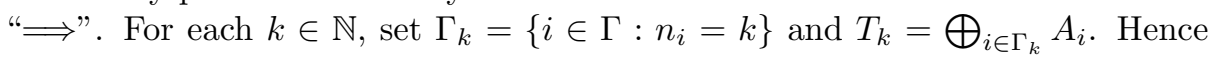
$T=\bigoplus_{k=1}^{\infty} T_{k}$ and, for each $k \in \mathbb{N}$, if $\Gamma_{k} \neq \emptyset$, then it is easy to check that $T_{k}$ is a regular nilpotent operator of order $k$.

Since $T$ is complex symmetric, it follows from Proposition 2.7 that $T_{k}$ is complex symmetric for all $k \in \mathbb{N}$. By Proposition 3.2, we conclude that $\operatorname{card}\{i \in \Gamma$ : $\left.\left(\left|\lambda_{1}^{(i)}\right|, \cdots,\left|\lambda_{n_{i}-1}^{(i)}\right|\right)=\mathbf{a}\right\}=\operatorname{card}\left\{i \in \Gamma:\left(\left|\lambda_{1}^{(i)}\right|, \cdots,\left|\lambda_{n_{i}-1}^{(i)}\right|\right)=\mathbf{a}^{t}\right\}$ for any $n \in \mathbb{N}$ and any $n$-tuple a.

Corollary 3.4. Assume that $T=\bigoplus_{i \in \Gamma} A_{i}$, where $1 \leq \operatorname{card} \Gamma \leq \aleph_{0}$,

$$
A_{i}=\left[\begin{array}{cccc}
0 & \lambda_{1}^{(i)} & & \\
& \ddots & \ddots & \\
& & 0 & \lambda_{n_{i}-1}^{(i)} \\
& & & 0
\end{array}\right] \begin{gathered}
e_{1}^{i} \\
\vdots \\
e_{n_{i}-1}^{i} \\
e_{n_{i}}^{i}
\end{gathered}
$$

with respect to some ONB $\left\{e_{j}^{i}\right\}_{j=1}^{n_{i}}$ of the underlying space of $A_{i}$ and nul $A_{i}=1$ for each $i \in \Gamma$. If $\Gamma_{1}$ is a nonempty finite subset of $\Gamma$ and $\bigoplus_{i \in \Gamma_{1}} A_{i}$ is complex symmetric, then $T \in(\mathrm{CS})$ if and only if $\bigoplus_{i \in \Gamma \backslash \Gamma_{1}} A_{i} \in(\mathrm{CS})$.

Proposition 3.5. Let $T$ be a unilateral weighted shift on $\mathcal{H}$ with weighted sequence $\left\{w_{i}\right\}_{i=1}^{\infty}$, where $\operatorname{dim} \mathcal{H}=\aleph_{0}$. If $T$ is complex symmetric, then card $\left\{i \in \mathbb{N}: w_{i}=\right.$ $0\}=\aleph_{0}$.

Proof. For a proof by contradiction, we assume $\operatorname{card}\left\{i \in \mathbb{N}: w_{i}=0\right\}<\infty$. In this case, it is easy to see that nul $T \neq \operatorname{nul} T^{*}$. Then, by Lemma 2.3. $T \notin(\mathrm{CS})$, a contradiction.

Remark 3.6. In view of Proposition 3.5. Theorem 3.3 actually gives a characterization of unilateral weighted shifts being complex symmetric.

Now we are going to give the proof of Theorem 3.1 .

Proof of Theorem 3.1. By Lemma 2.8, the sufficiency is obvious. We need only prove the necessity. 


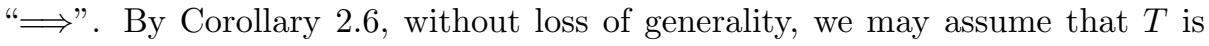
abnormal. By Proposition 3.5 and Theorem 3.3. $T \in(\mathrm{CS})$ implies that $T$ can be written as $T=\bigoplus_{i=1}^{\infty} A_{i}$, where

$$
A_{i}=\left[\begin{array}{cccc}
0 & \lambda_{1}^{(i)} & & \\
& \ddots & \ddots & \\
& & 0 & \lambda_{n_{i}-1}^{(i)} \\
& & & 0
\end{array}\right]
$$

with respect to some ONB of the underlying space of $A_{i}$ and nul $A_{i}=1$ for $i \in \mathbb{N}$; moreover, $\operatorname{card}\left\{i \in \mathbb{N}:\left(\left|\lambda_{1}^{(i)}\right|, \cdots,\left|\lambda_{n_{i}-1}^{(i)}\right|\right)=\mathbf{a}\right\}=\operatorname{card}\left\{i \in \mathbb{N}:\left(\left|\lambda_{1}^{(i)}\right|, \cdots,\left|\lambda_{n_{i}-1}^{(i)}\right|\right)\right.$ $\left.=\mathbf{a}^{t}\right\}$ for any $n \in \mathbb{N}$ and any $n$-tuple $\mathbf{a}$. Since $T$ is abnormal, we have $n_{i} \geq 2$ for all $i \in \mathbb{N}$.

Without loss of generality, we may directly assume that $\left|\lambda_{j}^{(i)}\right|=\lambda_{j}^{(i)}$ for all $i, j$. For $i \in \mathbb{N}$, denote $\mathbf{a}_{i}=\left(\lambda_{1}^{(i)}, \lambda_{2}^{(i)}, \cdots, \lambda_{n_{i}-1}^{(i)}\right)$. By our deduction, there exists a partition $\Delta=\left\{\Delta_{k}: k \in \mathbb{N}\right\}$ of $\mathbb{N}$ such that $1 \leq$ card $\Delta_{k} \leq 2$ for all $k$, and for each $k$,

(1) if card $\Delta_{k}=1$ and $i \in \Delta_{k}$, then $\mathbf{a}_{i}=\mathbf{a}_{i}^{t}$;

(2) if card $\Delta_{k}=2$ and $i, j \in \Delta_{k}, i \neq j$, then $\mathbf{a}_{i}=\mathbf{a}_{j}^{t}$.

Then, for each $k \in \mathbb{N}, T_{k}:=\bigoplus_{i \in \Delta_{k}} A_{i}$ has the form of

$$
\left[\begin{array}{cccc}
0 & \mu_{1} & & \\
& \ddots & \ddots & \\
& & 0 & \mu_{m_{k}-1} \\
& & & 0
\end{array}\right],
$$

where $m_{k} \in \mathbb{N}, m_{k} \geq 2$ and $\mu_{j}=\mu_{m_{k}-j}$ for all $1 \leq j \leq m_{k}-1$. Consequently, $T=\bigoplus_{k \in \mathbb{N}} T_{k}$. This completes the proof.

Remark 3.7. Let $T$ be the operator in Theorem 3.3. For each $i \in \Gamma$, it can be seen from the proof of Theorem 3.1 that if $T \in$ (CS), then either $A_{i} \in$ (CS) or there exists some $j \in \Gamma$ such that $A_{i} \oplus A_{j} \in(\mathrm{CS})$. Furthermore, this kind of corresponding relation between $i$ and $j$ induces a partition of $\Gamma$.

\section{Bilateral Case}

This section is devoted to characterizing bilateral weighted shifts with complex symmetry. The main result of this section is the following theorem, which implies that complex symmetric bilateral weighted shifts possess at most three possible forms.

Theorem 4.1. Let $T$ be a bilateral weighted shift on $\mathcal{H}$ with weighted sequence $\left\{w_{i}\right\}_{i \in \mathbb{Z}}$, where $\operatorname{dim} \mathcal{H}=\aleph_{0}$. Then $T$ is complex symmetric if and only if exactly one of the following three statements holds.

(i) $w_{i} \neq 0$ for all $i \in \mathbb{Z}$ and there exists $m \in \mathbb{Z}$ such that $\left|w_{i}\right|=\left|w_{m-i}\right|$ for all $i \in \mathbb{Z}$.

(ii) $T$ is unitarily equivalent to a complex symmetric unilateral weighted shift. 
(iii) $T \simeq A \oplus A^{*} \oplus B$, where $A$ is an injective unilateral weighted shift and $B$ is absent or $B=\bigoplus_{i=1}^{k} B_{i}, k \in \mathbb{N}$, each $B_{i}$ admits the following matrix representation

$$
B_{i}=\left[\begin{array}{ccccc}
0 & \lambda_{1}^{(i)} & & & \\
& 0 & \lambda_{2}^{(i)} & & \\
& & \ddots & \ddots & \\
& & & 0 & \lambda_{n_{i}-1}^{(i)} \\
& & & & 0
\end{array}\right]
$$

with respect to some ONB of the underlying space of $B_{i}$ and $\left|\lambda_{j}^{(i)}\right|=\left|\lambda_{n_{i}-j}^{(i)}\right|$ for $1 \leq j \leq n_{i}-1$.

For two operators $A, B$, we let $A \simeq B$ denote that $A, B$ are unitarily equivalent.

Lemma 4.2. Let $\left\{e_{i}\right\}_{i \in \mathbb{Z}}$ be an ONB of $\mathcal{H}$ and $T \in \mathcal{B}(\mathcal{H})$ with $T e_{i}=\alpha_{i} e_{i+1}$ for $i \in \mathbb{Z}$. Assume that $T$ is $C$-symmetric and $\alpha_{i}>0$ for all $i \in \mathbb{Z}$. Then

(i) $C e_{k} \in \bigvee\left\{e_{j}: \alpha_{j}=\alpha_{k-1}\right\}$ for all $k \in \mathbb{Z}$;

(ii) if $\Gamma \subset \mathbb{Z}, k \in \mathbb{Z}$ and $C e_{k} \in \bigvee\left\{e_{i}: i \in \Gamma\right\}$, then $C e_{k-j} \in \bigvee\left\{e_{i+j}: i \in \Gamma\right\}$ for all $j \in \mathbb{Z}$.

Proof. (i) Assume that $T=U|T|$ is the polar decomposition of $T$. It is easy to check that $U e_{j}=e_{j+1}$ and $|T| e_{j}=\alpha_{j} e_{j}$ for all $j \in \mathbb{Z}$. Since $T$ is $C$-symmetric, it follows from Lemma 2.1 that $C U|T|=|T| C U$. Then, given $k \in \mathbb{Z}$, we have

$$
\begin{aligned}
\alpha_{k-1} C e_{k} & =\alpha_{k-1} C U e_{k-1}=C U \alpha_{k-1} e_{k-1} \\
& =C U|T| e_{k-1}=|T| C U e_{k-1}=|T| C e_{k},
\end{aligned}
$$

that is, $C e_{k} \in \operatorname{ker}\left(|T|-\alpha_{k-1}\right)=\bigvee\left\{e_{j}: \alpha_{j}=\alpha_{k-1}\right\}$. This completes the proof.

(ii) Fix a $k \in \mathbb{Z}$. Assume that $C e_{k}=\sum_{i \in \Gamma} \beta_{i} e_{i}$. Then

$$
\begin{aligned}
\alpha_{k-1} C e_{k-1} & =C \alpha_{k-1} e_{k-1}=C T^{*} e_{k} \\
& =T C e_{k}=\sum_{i \in \Gamma} \beta_{i} T e_{i}=\sum_{i \in \Gamma} \beta_{i} \alpha_{i} e_{i+1}
\end{aligned}
$$

and

$$
\begin{aligned}
\alpha_{k} C e_{k+1} & =C \alpha_{k} e_{k+1}=C T e_{k} \\
& =T^{*} C e_{k}=\sum_{i \in \Gamma} \beta_{i} T^{*} e_{i}=\sum_{i \in \Gamma} \beta_{i} \alpha_{i-1} e_{i-1} .
\end{aligned}
$$

That is, $C e_{k-1} \in \bigvee\left\{e_{i+1}: i \in \Gamma\right\}$ and $C e_{k+1} \in \bigvee\left\{e_{i-1}: i \in \Gamma\right\}$. Using a recursive argument, one can see the conclusion.

Corollary 4.3. Let $\left\{e_{i}\right\}_{i \in \mathbb{Z}}$ be an ONB of $\mathcal{H}$ and $T \in \mathcal{B}(\mathcal{H})$ with $T e_{i}=\alpha_{i} e_{i+1}$ for $i \in \mathbb{Z}$. Assume that $T$ is $C$-symmetric and $\alpha_{i}>0$ for all $i \in \mathbb{Z}$. If $m, n \in \mathbb{Z}$ and $\left(C e_{m}, e_{n}\right) \neq 0$, then $\alpha_{m+n-1-j}=\alpha_{j}$ for all $j \in \mathbb{Z}$.

Proof. Since $\left(C e_{m}, e_{n}\right) \neq 0$, it can be seen from the proof of Lemma 4.2 (ii) that

$$
\left(C e_{m-j}, e_{n+j}\right) \neq 0, \forall j \in \mathbb{Z} .
$$

By Lemma 4.2 (i), it follows immediately that $\alpha_{m-1-j}=\alpha_{n+j}$ for all $j \in \mathbb{Z}$. This completes the proof. 
Theorem 4.4. Let $\left\{e_{i}\right\}_{i \in \mathbb{Z}}$ be an ONB of $\mathcal{H}$ and $T \in \mathcal{B}(\mathcal{H})$ with $T e_{i}=\alpha_{i} e_{i+1}$ for $i \in \mathbb{Z}$. If $\alpha_{i} \neq 0$ for all $i \in \mathbb{Z}$, then $T$ is complex symmetric if and only if there exists $k \in \mathbb{Z}$ such that $\left|\alpha_{k-j}\right|=\left|\alpha_{j}\right|$ for all $j \in \mathbb{Z}$.

Proof. Since $T$ is unitarily equivalent to the operator $\sum_{i \in \mathbb{Z}}\left|\alpha_{i}\right| e_{i+1} \otimes e_{i}$, we may directly assume that $\alpha_{i}>0$ for all $i \in \mathbb{Z}$.

"£". For $x=\sum_{i \in \mathbb{Z}} \beta_{i} e_{i} \in \mathcal{H}$, we define

$$
C: \sum_{i \in \mathbb{Z}} \beta_{i} e_{i} \mapsto \sum_{i \in \mathbb{Z}} \overline{\beta_{i}} e_{k+1-i} .
$$

Then $C$ is a conjugation on $\mathcal{H}$ and, for $j \in \mathbb{Z}$, we have

$$
\begin{aligned}
C T C e_{j} & =C T e_{k+1-j}=C \alpha_{k+1-j} e_{k+2-j} \\
& =\alpha_{k+1-j} e_{j-1}=\alpha_{j-1} e_{j-1}=T^{*} e_{j},
\end{aligned}
$$

that is, $C T C e_{j}=T^{*} e_{j}$. Thus $C T C=T^{*}$ and $T$ is $C$-symmetric.

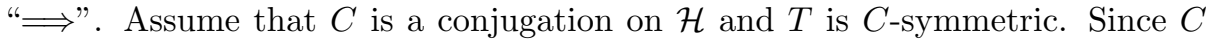
is invertible, $C e_{0} \neq 0$ and there exists some $n \in \mathbb{Z}$ such that $\left(C e_{0}, e_{n}\right) \neq 0$. Set $k=n-1$. Then, using Corollary 4.3, one can see that $\alpha_{k-j}=\alpha_{j}$ for all $j \in \mathbb{Z}$.

Note that the proof for the sufficiency of Theorem 4.4 has nothing to do with the condition " $\alpha_{i} \neq 0$ for all $i$ ". Thus the following result is obvious.

Corollary 4.5. Let $\left\{e_{i}\right\}_{i \in \mathbb{Z}}$ be an ONB of $\mathcal{H}$ and $T \in \mathcal{B}(\mathcal{H})$ with $T e_{i}=\alpha_{i} e_{i+1}$ for $i \in \mathbb{Z}$. If there exists $k \in \mathbb{Z}$ such that $\left|\alpha_{k-j}\right|=\left|\alpha_{j}\right|$ for all $j \in \mathbb{Z}$, then $T$ is complex symmetric.

Corollary 4.6. Let $A$ be a unilateral weighted shift on $\mathcal{H}$. Then $T=A \oplus A^{*}$ is complex symmetric on $\mathcal{H} \oplus \mathcal{H}$.

Proof. Without loss of generality, we assume that $\left\{e_{i}\right\}_{i \in \mathbb{N}}$ is an ONB of $\mathcal{H}$ and $A e_{i}=\alpha_{i} e_{i+1}$ for $i \in \mathbb{N}$. Set $B e_{i}=\left|\alpha_{i}\right| e_{i+1}$ for $i \in \mathbb{N}$. Then $B \oplus B^{*} \simeq T$ and $B \oplus B^{*}$ is unitarily equivalent to a bilateral weighted shift with weight sequence $\left\{w_{i}\right\}_{i \in \mathbb{Z}}$, where $w_{0}=0$ and $w_{i}=\left|\alpha_{i}\right|=w_{-i}$ for all $i \in \mathbb{N}$, that is, $w_{i}=w_{-i}$ for all $i \in \mathbb{N}$. Then, by Corollary 4.5, we conclude that $T$ is complex symmetric.

Remark 4.7. By Theorem 4.4 the complex symmetry of a bilateral weighted shift with nonzero weights means some kind of symmetry of its weighted sequence.

Theorem 4.8. Let $\left\{e_{i}\right\}_{i \in \mathbb{Z}}$ be an ONB of $\mathcal{H}$ and $T \in \mathcal{B}(\mathcal{H})$ with $T e_{i}=\alpha_{i} e_{i+1}$ for $i \in \mathbb{Z}$. If $0<\operatorname{card}\left\{i \in \mathbb{Z}: \alpha_{i}=0\right\}<\infty$, then $T$ is complex symmetric if and only if $T \simeq A \oplus A^{*} \oplus B$, where (1) $A$ is an injective unilateral weighted shift, and (2) $B$ may be absent or $B=\bigoplus_{i=1}^{k} B_{i}, k \in \mathbb{N}$, each $B_{i}$ admits the following matrix representation

$$
B_{i}=\left[\begin{array}{ccccc}
0 & \lambda_{1}^{(i)} & & & \\
& 0 & \lambda_{2}^{(i)} & & \\
& & \ddots & \ddots & \\
& & & 0 & \lambda_{n_{i}-1}^{(i)} \\
& & & & 0
\end{array}\right]
$$

with respect to some ONB of the underlying space of $B_{i}$ and $\left|\lambda_{j}^{(i)}\right|=\left|\lambda_{n_{i}-j}^{(i)}\right|$ for $1 \leq j \leq n_{i}-1$. 
Proof. In view of Corollary 4.6 and Lemma 2.8, the sufficiency is obvious. We need only prove the necessity.

Without loss of generality, we assume that $T$ is $C$-symmetric and $\alpha_{i}=\left|\alpha_{i}\right|$ for all $i \in \mathbb{Z}$.

Case 1. $\operatorname{card}\left\{i \in \mathbb{N}: \alpha_{i}=0\right\}=1$. In this case, we may also assume that $\alpha_{0}=0$. Then $\operatorname{ker} T^{m}=\bigvee\left\{e_{i}: 1-m \leq i \leq 0\right\}$ and $\operatorname{ker}\left(T^{*}\right)^{m}=\bigvee\left\{e_{i}: 1 \leq i \leq m\right\}$ for all $m \in \mathbb{N}$. Note that $C\left[\operatorname{ker}\left(T^{*}\right)^{m}\right]=\operatorname{ker} T^{m}$ for all $m \in \mathbb{N}$ and, for $x, y \in \mathcal{H}$, $(C x, C y)=0$ if and only if $(x, y)=0$. Then, for each $m \in \mathbb{N}, C\left(\bigvee\left\{e_{m}\right\}\right)=$ $\bigvee\left\{e_{1-m}\right\}$. On the other hand, $C$ preserves the norms of vectors. Then there exists $\lambda_{m} \in \mathbb{C}$ with $\left|\lambda_{m}\right|=1$ such that $C e_{m}=\lambda_{m} e_{1-m}$ for $m \in \mathbb{N}$. Hence

$$
\begin{aligned}
\alpha_{m-1} & =\left|C \alpha_{m-1} e_{m-1}\right|=\left|C T^{*} e_{m}\right| \\
& =\left|T C e_{m}\right|=\left|\lambda_{m} T e_{1-m}\right|=\left|\lambda_{m} \alpha_{1-m} e_{2-m}\right|=\alpha_{1-m}
\end{aligned}
$$

for all $m \in \mathbb{N}$. That is, $\alpha_{i}=\alpha_{-i}$ for all $i \in \mathbb{N}$. Choose another onB $\left\{f_{i}\right\}_{i=1}^{\infty}$ of $\mathcal{H}$ and define $A \in \mathcal{B}(\mathcal{H})$ as $A f_{i}=\alpha_{i} f_{i+1}$ for $i \geq 1$. Then it is obvious that $T \simeq A \oplus A^{*}$.

Case 2. $1<\operatorname{card}\left\{i \in \mathbb{N}: \alpha_{i}=0\right\}<\infty$. In this case, there exist $m, n \in \mathbb{Z}$, $m<n$, such that $\alpha_{n}=0=\alpha_{m}$ and $\alpha_{i} \neq 0$ for all $i>n$ or $i<m$. Denote $\mathcal{H}_{1}=\bigvee\left\{e_{i}: i \leq m\right\}, \mathcal{H}_{2}=\bigvee\left\{e_{i}: m<i \leq n\right\}$ and $\mathcal{H}_{3}=\bigvee\left\{e_{i}: i>n\right\}$. Then each $\mathcal{H}_{i}$ is a reducing subspace of $T$. For $1 \leq i \leq 3$, denote $T_{i}=\left.T\right|_{\mathcal{H}_{i}}$. Then $T_{1}^{*}$ and $T_{3}$ are two injective unilateral weighted shifts. Note that $T_{2}$ is nilpotent and $T_{2}^{k}=0$, where $k=n-m$.

Claim. $T_{2}$ and $T_{1} \oplus T_{3}$ are both complex symmetric.

Obviously, it suffices to prove that $C\left(\mathcal{H}_{2}\right)=\mathcal{H}_{2}$. Arbitrarily choose an $x \in \mathcal{H}_{2}$. Since $\mathcal{H}_{2}=\operatorname{ker} T_{2}^{k} \subset \operatorname{ker} T^{k}$, we have $T^{k} x=0$ and

$$
C x \in \operatorname{ker}\left(T^{k}\right)^{*}=\operatorname{ker}\left(T_{1}^{k}\right)^{*} \oplus \operatorname{ker}\left(T_{2}^{k}\right)^{*} \oplus \operatorname{ker}\left(T_{3}^{k}\right)^{*} .
$$

Since $\left(T_{1}^{k}\right)^{*}$ is injective and $T_{2}^{k}=0$, we obtain $C x \in \mathcal{H}_{2} \oplus \operatorname{ker}\left(T_{3}^{k}\right)^{*}$. On the other hand, we note that

$$
T^{k} C x=C\left(T^{k}\right)^{*} x=C\left(T_{2}^{k}\right)^{*} x=0 .
$$

Then $C x \in \operatorname{ker} T^{k}=\operatorname{ker} T_{1}^{k} \oplus \mathcal{H}_{2}$ and hence $C x \in \mathcal{H}_{2}$. Then $C\left(\mathcal{H}_{2}\right) \subset \mathcal{H}_{2}$, and it follows from $C^{2}=I$ that $C\left(\mathcal{H}_{2}\right)=\mathcal{H}_{2}$. This proves the claim.

By Theorem 3.3 and Case 1, it can be seen from the above claim that $T$ has the form as stated in the theorem.

Theorem 4.9. Let $\left\{e_{i}\right\}_{i \in \mathbb{Z}}$ be an ONB of $\mathcal{H}$ and $T \in \mathcal{B}(\mathcal{H})$ with $T e_{i}=\alpha_{i} e_{i+1}$ for $i \in \mathbb{Z}$. If $\operatorname{card}\left\{i \in \mathbb{Z}: \alpha_{i}=0\right\}=\infty$, then $T$ is complex symmetric if and only if $T \simeq \bigoplus_{i=1}^{\infty} T_{i}$, where each $T_{i}$ admits the following matrix representation:

$$
T_{i}=\left[\begin{array}{ccccc}
0 & \lambda_{1}^{(i)} & & & \\
& 0 & \lambda_{2}^{(i)} & & \\
& & \ddots & \ddots & \\
& & & 0 & \lambda_{n_{i}-1}^{(i)} \\
& & & & 0
\end{array}\right]
$$

with respect to some ONB of the underlying space of $T_{i}$ and $\left|\lambda_{j}^{(i)}\right|=\left|\lambda_{n_{i}-j}^{(i)}\right|$ for $1 \leq j \leq n_{i}-1$. 
Proof. By Lemma 2.8 the sufficiency is obvious. We need only prove the necessity.

"£". Denote $\Gamma=\left\{i \in \mathbb{Z}: \alpha_{i}=0\right\}$. It suffices to prove that $\Gamma$ has neither upper nor lower bound. In fact, if this holds, then, by rearranging the vectors in the ONB $\left\{e_{i}\right\}_{i \in \mathbb{Z}}$, one can see that $T$ is also a unilateral weighted shift. Then, by Theorem 3.1, one can obtain the conclusion.

For a proof by contradiction, we may directly assume that $\sup \Gamma<+\infty$ and $n=\sup \Gamma$ (the proof for the case "inf $\Gamma>-\infty$ " is similar). In this case, $T$ can be written as

$$
T=\left(\bigoplus_{i=1}^{\infty} A_{i}\right) \oplus B
$$

where each $A_{i}$ is nilpotent and $B$ is an injective unilateral weighted shift. Since nul $B=0$ and nul $B^{*}=1, B$ is not complex symmetric.

Denote by $\mathcal{H}_{i}$ and $\mathcal{K}$ the underlying spaces of $A_{i}$ and $B$ respectively for $i \in \mathbb{N}$. Arbitrarily choose an $i \in \mathbb{N}$ and an $x \in \mathcal{H}_{i}$. Assume that $A_{i}^{k_{i}}=0$ and $T$ is $C$ symmetric. Then $0=C\left(A_{i}^{k_{i}}\right)^{*} x=C\left(T^{k_{i}}\right)^{*} x=T^{k_{i}} C x$ and hence $C x \in \operatorname{ker} T^{k_{i}} \subset$ $\bigoplus_{i=1}^{\infty} \mathcal{H}_{i}$. Since $i \in \mathbb{N}$ and $x \in \mathcal{H}_{i}$ were arbitrarily chosen, we have $C\left(\bigoplus_{i=1}^{\infty} \mathcal{H}_{i}\right) \subset$ $\left(\bigoplus_{i=1}^{\infty} \mathcal{H}_{i}\right)$. Note that $C^{2}=I$. Then $C\left(\bigoplus_{i=1}^{\infty} \mathcal{H}_{i}\right)=\bigoplus_{i=1}^{\infty} \mathcal{H}_{i}$ and $C(\mathcal{K})=\mathcal{K}$. Set $C_{1}=\left.C\right|_{\mathcal{K}}$. Then it is easy to verify that $C_{1}$ is a conjugation on $\mathcal{K}$ and $B$ is $C_{1}$-symmetric, a contradiction.

Remark 4.10. Summarizing the results of Theorems 4.4, 4.8 and 4.9, one can obtain Theorem 4.1

\section{Generalized Aluthge transforms of weighted shifts}

This section is devoted to characterizing those (unilateral or bilateral) weighted shifts $T$ satisfying that $T_{\varepsilon} \in(\mathrm{CS})$ for all $\varepsilon \in[0,1]$. The main results of this section are Theorems [5.11, 5.13 and 5.15.

Lemma 5.1. Let $n \in \mathbb{N}$ and $n \geq 3$. Assume that $\left\{e_{i}\right\}_{i=1}^{n}$ is an ONB of $\mathbb{C}^{n}$ and $T=\sum_{i=1}^{n-1} \lambda_{i} e_{i} \otimes e_{i+1}$. If $\varepsilon \in(0,1]$, then

$$
T \simeq \sum_{i=1}^{n-1}\left|\lambda_{i}\right| e_{i} \otimes e_{i+1}, \quad T_{\varepsilon} \simeq \sum_{i=2}^{n-1}\left|\lambda_{i-1}^{\varepsilon} \lambda_{i}^{1-\varepsilon}\right| e_{i} \otimes e_{i+1}
$$

and $T_{\varepsilon}^{n-1}=0$.

By Lemma 5.1 and Theorem 3.3 the following corollary is clear.

Corollary 5.2. Let $n \in \mathbb{N}$ and $n \geq 4$ be odd. Assume that $\left\{e_{i}\right\}_{i=1}^{n}$ is an ONB of $\mathbb{C}^{n}$ and $T=\sum_{i=1}^{n-1} \lambda_{i} e_{i} \otimes e_{i+1}$. If $\left|\lambda_{i}\right|=\left|\lambda_{i+2}\right|$ for all $1 \leq i \leq n-3$, then $T_{\varepsilon} \in(\mathrm{CS})$ for all $\varepsilon \in(0,1]$.

Proposition 5.3. Let $n \in \mathbb{N}, n \geq 2$ and $\left\{e_{j}\right\}_{j=1}^{n}$ be an ONB of $\mathbb{C}^{n}$. Assume that $T \in \mathcal{B}\left(\mathbb{C}^{n}\right), T=\sum_{i=1}^{n-1} \lambda_{i} e_{i} \otimes e_{i+1}$ and $\lambda_{i} \neq 0$ for all $1 \leq i \leq n-1$. Then the following are equivalent:

(i) $T_{\varepsilon} \in(\mathrm{CS})$ for all $\varepsilon \in[0,1]$;

(ii) $T_{\varepsilon} \in(\mathrm{CS})$ for all $\varepsilon \in\left[0, \frac{1}{2}\right]$;

(iii) $T \in(\mathrm{CS})$ and there exists $\varepsilon \in\left(0, \frac{1}{2}\right)$ such that $T_{\varepsilon} \in(\mathrm{CS})$;

(iv) $\left|\lambda_{1}\right|=\left|\lambda_{2}\right|=\cdots=\left|\lambda_{n-1}\right|$. 
Proof. The relations "(i) $\Rightarrow($ ii $) \Rightarrow($ iii)" and "(iv) $\Rightarrow(\mathrm{i})$ " are obvious. We need only prove "(iii) $\Rightarrow$ (iv)". When $n=2$, the relation is obvious. In the following, we assume that $n \geq 3$ and, without loss of generality, assume that $\lambda_{i}>0$ for all $1 \leq i \leq n-1$. Note that

$$
T_{\varepsilon}=\left[\begin{array}{ccccc}
0 & 0 & & & \\
& 0 & \mu_{1} & & \\
& & \ddots & \ddots & \\
& & & 0 & \mu_{n-2} \\
& & & & 0
\end{array}\right] \begin{gathered}
e_{1} \\
e_{2} \\
\vdots \\
e_{n-1} \\
e_{n}
\end{gathered}
$$

where $\mu_{i}=\lambda_{i}^{\varepsilon} \lambda_{i+1}^{1-\varepsilon}$ for $1 \leq i \leq n-2$. By Corollary 2.6 and Theorem 3.3. $T \in$ (CS) and $T_{\varepsilon} \in$ (CS) imply that $\lambda_{i}=\lambda_{n-i}$ for $1 \leq i \leq n-1$ and $\lambda_{i}^{\varepsilon} \lambda_{i+1}^{1-\varepsilon}=\lambda_{n-i-1}^{\varepsilon} \lambda_{n-i}^{1-\varepsilon}$ for $1 \leq i \leq n-2$. Furthermore, it follows that $\lambda_{i}^{\varepsilon} \lambda_{i+1}^{1-\varepsilon}=\lambda_{i+1}^{\varepsilon} \lambda_{i}^{1-\varepsilon}$ for $1 \leq i \leq n-2$. Since $0<\varepsilon<1 / 2$, we deduce that $\lambda_{i}=\lambda_{i+1}$ for $1 \leq i \leq n-2$, that is, $\lambda_{1}=\lambda_{2}=$ $\cdots=\lambda_{n-1}$.

Example 5.4. Let $\left\{e_{i}\right\}_{i=1}^{4}$ be an ONB of $\mathbb{C}^{4}$ and set $T=e_{1} \otimes e_{2}+2 e_{2} \otimes e_{3}+e_{3} \otimes e_{4}$. By Lemma 2.8 and Proposition 5.3. $T \in(\mathrm{CS})$ and $T_{\varepsilon} \notin(\mathrm{CS})$ for all $\varepsilon \in(0,1 / 2)$. Thus we give a negative answer to Question 1.3 .

Proposition 5.5. Let $n \in \mathbb{N}, n \geq 4$ and $\left\{e_{j}\right\}_{j=1}^{n}$ be an ONB of $\mathbb{C}^{n}$. Assume that $A \in \mathcal{B}\left(\mathbb{C}^{n}\right)$ admits the following matrix representation:

$$
A=\left[\begin{array}{cccc}
0 & \lambda_{1} & & \\
& \ddots & \ddots & \\
& & 0 & \lambda_{n-1} \\
& & & 0
\end{array}\right] \begin{gathered}
e_{1} \\
\vdots \\
e_{n-1} \\
e_{n}
\end{gathered}
$$

and nul $A=1$. If there exist two distinct $\varepsilon_{1}, \varepsilon_{2} \in(0,1 / 2)$ such that $A_{\varepsilon_{j}} \in(\mathrm{CS})$ for $j=1,2$, then $\left|\lambda_{i}\right|=\left|\lambda_{i+2}\right|$ for $1 \leq i \leq n-3$.

Proof. For $1 \leq j \leq 2$, since $A_{\varepsilon_{j}} \in(\mathrm{CS})$, it follows that $\left|\lambda_{i}^{\varepsilon_{j}} \lambda_{i+1}^{1-\varepsilon_{j}}\right|=\left|\lambda_{n-i-1}^{\varepsilon_{j}} \lambda_{n-i}^{1-\varepsilon_{j}}\right|$ for $1 \leq i \leq n-2$. Thus we have

$$
\left|\frac{\lambda_{i} \lambda_{n-i}}{\lambda_{n-i-1} \lambda_{i+1}}\right|^{\varepsilon_{j}}=\left|\frac{\lambda_{n-i}}{\lambda_{i+1}}\right|, \quad \forall 1 \leq j \leq 2,1 \leq i \leq n-2 .
$$

Noting that $\varepsilon_{1} \neq \varepsilon_{2}$, one can see that $\left|\lambda_{n-i}\right|=\left|\lambda_{i+1}\right|$ and $\left|\lambda_{n-i-1}\right|=\left|\lambda_{i}\right|$ for $1 \leq i \leq n-2$. A simple calculation shows that $\left|\lambda_{i}\right|=\left|\lambda_{i+2}\right|$ for $1 \leq i \leq n-3$.

Proposition 5.6. Let $n \in \mathbb{N}, n \geq 4$ and $\left\{e_{i}\right\}_{i=1}^{n}$ be an ONB of $\mathbb{C}^{n}$. Assume that $A, B \in \mathcal{B}\left(\mathbb{C}^{n}\right)$ admit the following matrix representations:

$$
A=\left[\begin{array}{cccc}
0 & \lambda_{1} & & \\
& \ddots & \ddots & \\
& & 0 & \lambda_{n-1} \\
& & & 0
\end{array}\right] \begin{gathered}
e_{1} \\
\vdots \\
e_{n-1} \\
e_{n}
\end{gathered}, \quad B=\left[\begin{array}{cccc}
0 & \mu_{1} & & \\
& \ddots & \ddots & \\
& & 0 & \mu_{n-1} \\
& & & 0
\end{array}\right] \begin{gathered}
e_{1} \\
\vdots \\
e_{n-1} \\
e_{n}
\end{gathered}
$$

and nul $A=1=\operatorname{nul} B$. If there exist two distinct $\varepsilon_{1}, \varepsilon_{2} \in(0,1 / 2)$ such that (1) $A_{\varepsilon_{j}} \oplus B_{\varepsilon_{j}} \in(\mathrm{CS})$ for $j=1,2$, and (2) $A_{\varepsilon_{j}} \notin(\mathrm{CS})$ for $j=1,2$, then $n-1$ is odd, $\left|\lambda_{1}\right| \neq\left|\lambda_{2}\right|$ and $\left|\lambda_{i}\right|=\left|\mu_{j}\right|$ when $i+j$ is odd. 
Proof. For $1 \leq j \leq 2$, since $A_{\varepsilon_{j}} \notin$ (CS) and $A_{\varepsilon_{j}} \oplus B_{\varepsilon_{j}} \in$ (CS), by Corollary 2.6. Lemma 5.1 and Theorem 3.3, it follows that $\left|\lambda_{i}^{\varepsilon_{j}} \lambda_{i+1}^{1-\varepsilon_{j}}\right|=\left|\mu_{n-i-1}^{\varepsilon_{j}} \mu_{n-i}^{1-\varepsilon_{j}}\right|$ for $1 \leq i \leq n-2$. Thus we have

$$
\left|\frac{\lambda_{i} \mu_{n-i}}{\mu_{n-i-1} \lambda_{i+1}}\right|^{\varepsilon_{j}}=\left|\frac{\mu_{n-i}}{\lambda_{i+1}}\right|, \quad \forall 1 \leq j \leq 2,1 \leq i \leq n-2 .
$$

Noting that $\varepsilon_{1} \neq \varepsilon_{2}$, one can see that $\left|\mu_{n-i}\right|=\left|\lambda_{i+1}\right|$ and $\left|\mu_{n-i-1}\right|=\left|\lambda_{i}\right|$ for $1 \leq i \leq n-2$. A simple calculation shows that $\left|\lambda_{1}\right|=\left|\mu_{n-2}\right|,\left|\lambda_{2}\right|=\left|\mu_{n-1}\right|$, $\left|\lambda_{i}\right|=\left|\lambda_{i+2}\right|$ and $\left|\mu_{i}\right|=\left|\mu_{i+2}\right|$ for $1 \leq i \leq n-3$.

It remains to prove that $n-1$ is odd and $\left|\lambda_{1}\right| \neq\left|\lambda_{2}\right|$. In fact, if $n-1$ is even, then, by Corollary [5.2, $A_{\varepsilon} \in(\mathrm{CS})$ for all $0<\varepsilon<1$, a contradiction; if $\left|\lambda_{1}\right|=\left|\lambda_{2}\right|$, then $\left|\lambda_{1}\right|=\left|\lambda_{i}\right|$ for all $1 \leq i \leq n-1$ and, using Lemma 5.1, we have $A_{\varepsilon} \in$ (CS) for all $0<\varepsilon<1$, a contradiction.

Proposition 5.7. Let $n \in \mathbb{N}, n \leq 3$ and $T \in \mathcal{B}(\mathcal{H})$ be a unilateral weighted shift with weighted sequence $\left\{w_{i}\right\}_{i \in \mathbb{N}}$, where $w_{i}=0$ if and only if $n$ divides $i$. Then the following are equivalent:

(i) $T_{\varepsilon} \in(\mathrm{CS})$ for all $\varepsilon \in[0,1]$;

(ii) $T_{\varepsilon} \in(\mathrm{CS})$ for all $\varepsilon \in\left[0, \frac{1}{2}\right]$;

(iii) $T \in(\mathrm{CS})$.

Proof. Since $n \leq 3$, a simple calculation shows that $T_{\varepsilon}^{2}=0$ for all $\varepsilon \in(0,1]$. Then, by Lemma 2.2, $T_{\varepsilon} \in(\mathrm{CS})$ for all $\varepsilon \in(0,1]$. In view of this fact, the conclusion is obvious.

Proposition 5.8. Assume that $T=\bigoplus_{i \in \Gamma} T_{i}$, where $1 \leq \operatorname{card} \Gamma \leq \aleph_{0}$,

$$
T_{i}=\left[\begin{array}{cccc}
0 & \lambda_{1}^{(i)} & & \\
& \ddots & \ddots & \\
& & 0 & \lambda_{n_{i}-1}^{(i)} \\
& & & 0
\end{array}\right] \begin{gathered}
e_{1}^{i} \\
\vdots \\
e_{n_{i}-1}^{i} \\
e_{n_{i}}^{i}
\end{gathered}
$$

with respect to some ONB $\left\{e_{j}^{i}\right\}_{j=1}^{n_{i}}$ of the underlying space of $T_{i}$ and nul $T_{i}=1$ for each $i \in \Gamma$. If $T_{\varepsilon} \in(\mathrm{CS})$ for all $\varepsilon \in(0,1 / 2)$, then $\left|\lambda_{j}^{(i)}\right|=\left|\lambda_{j+2}^{(i)}\right|$ for all $i \in \Gamma$ with $n_{i} \geq 4$ and $1 \leq j \leq n_{i}-3$.

Proof. By Lemma 5.1, we may directly assume that $\left|\lambda_{j}^{(i)}\right|=\lambda_{j}^{(i)}$ for all $i, j$. Let $i \in \Gamma$ be fixed and assume that $n_{i} \geq 4$.

Case 1. $\operatorname{card}\left\{\varepsilon \in\left(0, \frac{1}{2}\right):\left(T_{i}\right)_{\varepsilon} \in(\mathrm{CS})\right\}=\operatorname{card}[0,1]$.

In this case, there exist two distinct $\varepsilon_{1}, \varepsilon_{2} \in\left(0, \frac{1}{2}\right)$ such that $\left(T_{i}\right)_{\varepsilon_{j}} \in(\mathrm{CS})$ for $j=1,2$. In view of Proposition 5.5 the above assumption implies that $\lambda_{j}^{(i)}=\lambda_{j+2}^{(i)}$ for $1 \leq j \leq n_{i}-3$.

Case 2. $\operatorname{card}\left\{\varepsilon \in\left(0, \frac{1}{2}\right):\left(T_{i}\right)_{\varepsilon} \notin(\mathrm{CS})\right\}=\operatorname{card}[0,1]$.

Denote $E=\left\{\varepsilon \in\left(0, \frac{1}{2}\right):\left(T_{i}\right)_{\varepsilon} \notin(\mathrm{CS})\right\}$. By Theorem 3.3 and Remark 3.7 . for each $\varepsilon \in E$, there exists $k_{\varepsilon} \in \Gamma, k_{\varepsilon} \neq i$, such that $\left(T_{k_{\varepsilon}}\right)_{\varepsilon} \oplus\left(T_{i}\right)_{\varepsilon}$ is complex symmetric. Using a cardinality analysis, there exist two distinct $\varepsilon_{1}, \varepsilon_{2} \in\left(0, \frac{1}{2}\right)$ such that $k_{\varepsilon_{1}}=k_{\varepsilon_{2}}$. Denote $k=k_{\varepsilon_{1}}=k_{\varepsilon_{2}}$. 
Since $\left(T_{k}\right)_{\varepsilon_{j}} \oplus\left(T_{i}\right)_{\varepsilon_{j}} \in(\mathrm{CS})$ and $\left(T_{i}\right)_{\varepsilon_{j}} \notin(\mathrm{CS})$ for $j=1,2$, it follows from Proposition 5.6 that $\lambda_{j}^{(i)}=\lambda_{j+2}^{(i)}$ for $1 \leq j \leq n_{i}-3$.

Let $n \in \mathbb{N}, n \geq 2$ and $\mathbf{a}=\left(a_{1}, a_{2}, \cdots, a_{n}\right)$ be an $n$-tuple of complex numbers. If $n$ is odd, then we denote $\mathbf{a}^{s}=\left(b_{1}, b_{2}, \cdots, b_{n}\right)$, where $b_{n}=a_{2}$ and $b_{i}=a_{i+1}$ for all $1 \leq i \leq n-1$; if $n$ is even, then we denote $\mathbf{a}^{s}=\left(c_{1}, c_{2}, \cdots, c_{n}\right)$, where $c_{n}=a_{1}$ and $c_{i}=a_{i+1}$ for all $1 \leq i \leq n-1$. For example, $(1,2,1,2,1)^{s}=(2,1,2,1,2)$ and $(1,2,1,2)^{s}=(2,1,2,1)$.

Using Lemma 5.1 and Theorem 3.3. one can easily verify the following result.

Lemma 5.9. Let $n \in \mathbb{N}$ be even and $n \geq 4$. Assume that $\left\{e_{i}\right\}_{i=1}^{n}$ is an ONB of $\mathbb{C}^{n}$, $A=\sum_{i=1}^{n-1} \lambda_{i} e_{i} \otimes e_{i+1}$ and $B=\sum_{i=1}^{n-1} \mu_{i} e_{i} \otimes e_{i+1}$. If $T=A \oplus B, \lambda_{i}=\lambda_{i+2}$ for all $1 \leq i \leq n-3$ and $\left(\mu_{1}, \mu_{2}, \cdots, \mu_{n-1}\right)=\left(\lambda_{1}, \lambda_{2}, \cdots, \lambda_{n-1}\right)^{s}$, then $T_{\varepsilon} \in(\mathrm{CS})$ for all $\varepsilon \in[0,1]$.

Proposition 5.10. Let $n \in \mathbb{N}$ and $n \geq 4$. Assume that $T=\bigoplus_{i \in \Gamma} T_{i}$, where $1 \leq \operatorname{card} \Gamma \leq \aleph_{0}$,

$$
T_{i}=\left[\begin{array}{cccc}
0 & \lambda_{1}^{(i)} & & \\
& \ddots & \ddots & \\
& & 0 & \lambda_{n-1}^{(i)} \\
& & & 0
\end{array}\right] \begin{gathered}
e_{1}^{i} \\
\vdots \\
e_{n-1}^{i} \\
e_{n}^{i}
\end{gathered}
$$

with respect to some ONB $\left\{e_{j}^{i}\right\}_{j=1}^{n}$ of the underlying space of $T_{i}$ and nul $T_{i}=1$ for each $i \in \Gamma$. Then the following are equivalent.

(i) $T_{\varepsilon} \in(\mathrm{CS})$ for all $\varepsilon \in[0,1]$.

(ii) $T_{\varepsilon} \in$ (CS) for all $\varepsilon \in\left[0, \frac{1}{2}\right]$.

(iii) $\left|\lambda_{j}^{(i)}\right|=\left|\lambda_{j+2}^{(i)}\right|$ for all $i \in \Gamma, 1 \leq j \leq n-3$ and $\operatorname{card}\left\{i \in \Gamma:\left(\left|\lambda_{1}^{(i)}\right|,\left|\lambda_{2}^{(i)}\right|, \cdots\right.\right.$, $\left.\left.\left|\lambda_{n-1}^{(i)}\right|\right)=\mathbf{a}\right\}=\operatorname{card}\left\{i \in \Gamma:\left(\left|\lambda_{1}^{(i)}\right|,\left|\lambda_{2}^{(i)}\right|, \cdots,\left|\lambda_{n-1}^{(i)}\right|\right)=\mathbf{a}^{s}\right\}$ for any $(n-1)-$ tuple $\mathbf{a}$.

Proof. Without loss of generality, we may directly assume that $\left|\lambda_{j}^{(i)}\right|=\lambda_{j}^{(i)}$ for all $i, j$. For each $i \in \Gamma$, denote $\mathbf{a}_{i}=\left(\lambda_{1}^{(i)}, \lambda_{2}^{(i)}, \ldots, \lambda_{n-1}^{(i)}\right)$.

"(i) $\Rightarrow$ (ii)". This is obvious.

"(ii) $\Rightarrow$ (iii)". Since $T_{\varepsilon} \in(\mathrm{CS})$ for all $\varepsilon \in\left(0, \frac{1}{2}\right)$, it follows from Proposition 5.8 that $\lambda_{j}^{(i)}=\lambda_{j+2}^{(i)}$ for all $i \in \Gamma$ and $1 \leq j \leq n-3$. Hence $\left(\mathbf{a}_{i}^{s}\right)^{s}=\mathbf{a}_{i}$ for all $i \in \Gamma$. Then it suffices to prove that $\operatorname{card}\left\{j \in \Gamma: \mathbf{a}_{j}=\mathbf{a}_{i}\right\}=\operatorname{card}\left\{j \in \Gamma: \mathbf{a}_{j}=\mathbf{a}_{i}^{s}\right\}$ for each $i \in \Gamma$.

Now we fix an $i \in \Gamma$.

Case 1. $\mathbf{a}_{i}^{t}=\mathbf{a}_{i}^{s}$.

By Theorem 3.3. $T \in(\mathrm{CS})$ implies that $\operatorname{card}\left\{j \in \Gamma: \mathbf{a}_{j}=\mathbf{a}\right\}=\operatorname{card}\{j \in \Gamma:$ $\left.\mathbf{a}_{j}=\mathbf{a}^{t}\right\}$ for all $(n-1)$-tuples $\mathbf{a}$. Then it is obvious that $\operatorname{card}\left\{j \in \Gamma: \mathbf{a}_{j}=\mathbf{a}_{i}\right\}=$ $\operatorname{card}\left\{j \in \Gamma: \mathbf{a}_{j}=\mathbf{a}_{i}^{s}\right\}$.

Case 2. $\mathbf{a}_{i}^{t} \neq \mathbf{a}_{i}^{s}$. In this case, it is easy to verify that $n-1$ is odd and there exist $a, b>0$ with $a \neq b$ such that $\mathbf{a}_{i}=(a, b, a, \cdots, b, a)$. Arbitrarily choosing an $\varepsilon \in\left(0, \frac{1}{2}\right)$, it is obvious that $\left(T_{i}\right)_{\varepsilon} \notin(\mathrm{CS})$. Since $T_{\varepsilon} \in(\mathrm{CS})$, by Theorem 3.3 , there exists $k \in \Gamma$ such that $\left(T_{i}\right)_{\varepsilon} \oplus\left(T_{k}\right)_{\varepsilon} \in(\mathrm{CS})$. By hypothesis, we may also assume that $\mathbf{a}_{k}=(c, d, c, \cdots, d, c)$ for $c, d>0$. Since $\left(T_{i}\right)_{\varepsilon} \oplus\left(T_{k}\right)_{\varepsilon} \in(\mathrm{CS})$, it follows from 
Lemma 5.1 and Theorem 3.3 that $a=d$ and $b=c$, and hence $\mathbf{a}_{i}=\mathbf{a}_{k}^{s}$. Also we note that $\mathbf{a}_{i} \neq \mathbf{a}_{i}^{s}$.

By the above argument, we claim that $\operatorname{card}\left\{j \in \Gamma: \mathbf{a}_{j}=\mathbf{a}_{i}\right\}=\operatorname{card}\left\{j \in \Gamma: \mathbf{a}_{j}=\right.$ $\left.\mathbf{a}_{i}^{s}\right\}$. For a proof by contradiction, we may assume that $\operatorname{card}\left\{j \in \Gamma: \mathbf{a}_{j}=\mathbf{a}_{i}\right\}<$ $\operatorname{card}\left\{j \in \Gamma: \mathbf{a}_{j}=\mathbf{a}_{i}^{s}\right\}$. Denote $\Gamma_{1}=\left\{j \in \Gamma: \mathbf{a}_{j}=\mathbf{a}_{i}\right\}$ and $\Gamma_{2}=\left\{j \in \Gamma: \mathbf{a}_{j}=\mathbf{a}_{i}^{s}\right\}$. Then $1 \leq \operatorname{card} \Gamma_{1}<\infty$ and $\Gamma_{1} \cap \Gamma_{2}=\emptyset$. We choose a subset $\Gamma_{2,1}$ of $\Gamma_{2}$ such that $\operatorname{card} \Gamma_{1}=\operatorname{card} \Gamma_{2,1}$. Set

$$
B_{1}=\bigoplus_{j \in \Gamma_{1} \cup \Gamma_{2,1}} T_{j}, \quad B_{2}=\bigoplus_{j \in \Gamma \backslash\left(\Gamma_{1} \cup \Gamma_{2,1}\right)} T_{j} .
$$

Then, by Lemma 5.9 and our above argument, $\left(B_{1}\right)_{\varepsilon} \in(\mathrm{CS})$. It follows from Corollary 3.4 that $\left(B_{2}\right)_{\varepsilon} \in(\mathrm{CS})$. Note that there exists no $j \in \Gamma \backslash\left(\Gamma_{1} \cup \Gamma_{2,1}\right)$ such that $\mathbf{a}_{j}=\mathbf{a}_{i}$.

Since $\Gamma_{2} \backslash \Gamma_{2,1} \neq \emptyset$, we can choose $j_{0} \in \Gamma_{2} \backslash \Gamma_{2,1}$. Then $\mathbf{a}_{i}=\mathbf{a}_{j_{0}}^{s}$. By our above argument, $\left(B_{2}\right)_{\varepsilon} \in(\mathrm{CS})$ implies that there exists $j_{1} \in \Gamma \backslash\left(\Gamma_{1} \cup \Gamma_{2,1}\right)$ such that $\mathbf{a}_{j_{0}}=\mathbf{a}_{j_{1}}^{s}$, that is, $\mathbf{a}_{i}=\mathbf{a}_{j_{0}}^{s}=\mathbf{a}_{j_{1}}$, a contradiction.

Thus we conclude that $\operatorname{card}\left\{j \in \Gamma: \mathbf{a}_{j}=\mathbf{a}_{i}\right\}=\operatorname{card}\left\{j \in \Gamma: \mathbf{a}_{j}=\mathbf{a}_{i}^{s}\right\}$.

"(iii) $\Rightarrow$ (i)". By condition (iii), there exists a partition $\Delta=\left\{\Delta_{k}: k \in \Lambda\right\}$ of $\Gamma$ such that $1 \leq \operatorname{card} \Delta_{k} \leq 2$ for all $k \in \Lambda$ and, for each $k \in \Lambda$,

(1) if card $\Delta_{k}=1$ and $i \in \Delta_{k}$, then $\mathbf{a}_{i}=\mathbf{a}_{i}^{s}$;

(2) if card $\Delta_{k}=2$ and $i_{1}, i_{2} \in \Delta_{k}, i_{1} \neq i_{2}$, then $\mathbf{a}_{i_{1}}=\mathbf{a}_{i_{2}}^{s}$.

For $k \in \Lambda$, set $A_{k}=\bigoplus_{i \in \Delta_{k}} T_{i}$.

The proof for the remaining part is divided into two cases.

Case 1. $n$ is odd. In this case, it is easy to see that $\mathbf{a}_{i}^{s}=\mathbf{a}_{i}^{t}$ for all $i \in \Gamma$.

By Lemma 2.8, $A_{k} \in(\mathrm{CS})$ for all $k$. Moreover, it follows from Corollary 5.2 that $\left(A_{k}\right)_{\varepsilon} \in(\mathrm{CS})$ for all $\varepsilon \in(0,1]$ and all $k \in \Lambda$. Hence $T_{\varepsilon}=\bigoplus_{k \in \Lambda}\left(A_{k}\right)_{\varepsilon} \in$ (CS) for all $\varepsilon \in[0,1]$.

Case 2. $n$ is even.

Let $k \in \Lambda$ be fixed. If card $\Delta_{k}=1$ and $i \in \Delta_{k}$, then it follows from $\mathbf{a}_{i}=\mathbf{a}_{i}^{s}$ that $\lambda_{1}^{(i)}=\lambda_{2}^{(i)}=\ldots=\lambda_{n-1}^{(i)}$. Then it is easily verified that $\left(A_{k}\right)_{\varepsilon} \in$ (CS) for all $\varepsilon \in[0,1]$. If card $\Delta_{k}=2$ and $i_{1}, i_{2} \in \Delta_{k}, i_{1} \neq i_{2}$, then $\mathbf{a}_{i_{1}}=\mathbf{a}_{i_{2}}^{s}$. By Lemma 5.9. this implies that $\left(A_{k}\right)_{\varepsilon} \in(\mathrm{CS})$ for all $\varepsilon \in[0,1]$. Thus $T_{\varepsilon}=\bigoplus_{k \in \Lambda}\left(A_{k}\right)_{\varepsilon} \in$ (CS) for all $\varepsilon \in[0,1]$.

Theorem 5.11. Assume that $T=\bigoplus_{i \in \Gamma} T_{i}$, where $1 \leq \operatorname{card} \Gamma \leq \aleph_{0}$,

$$
T_{i}=\left[\begin{array}{cccc}
0 & \lambda_{1}^{(i)} & & \\
& \ddots & \ddots & \\
& & 0 & \lambda_{n_{i}-1}^{(i)} \\
& & & 0
\end{array}\right] \begin{gathered}
e_{1}^{i} \\
\vdots \\
e_{n_{i}-1}^{i} \\
e_{n_{i}}^{i}
\end{gathered}
$$

with respect to some ONB $\left\{e_{j}^{i}\right\}_{j=1}^{n_{i}}$ of the underlying space of $T_{i}$ and nul $T_{i}=1$ for each $i \in \Gamma$. Then the following are equivalent.

(i) $T_{\varepsilon} \in(\mathrm{CS})$ for all $\varepsilon \in[0,1]$.

(ii) $T_{\varepsilon} \in$ (CS) for all $\varepsilon \in\left[0, \frac{1}{2}\right]$. 
(iii) $\left|\lambda_{j}^{(i)}\right|=\left|\lambda_{j+2}^{(i)}\right|$ for all $i \in \Gamma$ with $n_{i} \geq 4$ and $1 \leq j \leq n_{i}-3$, and $\operatorname{card}\{i \in \Gamma$ : $\left.\left(\left|\lambda_{1}^{(i)}\right|,\left|\lambda_{2}^{(i)}\right|, \cdots,\left|\lambda_{n_{i}-1}^{(i)}\right|\right)=\mathbf{a}\right\}=\operatorname{card}\left\{i \in \Gamma:\left(\left|\lambda_{1}^{(i)}\right|,\left|\lambda_{2}^{(i)}\right|, \cdots,\left|\lambda_{n_{i}-1}^{(i)}\right|\right)=\right.$ $\left.\mathbf{a}^{s}\right\}$ for any $n \in \mathbb{N}$ with $n \geq 2$ and any $n$-tuple $\mathbf{a}$.

Proof. For $k \in \mathbb{N}$, set $\Gamma_{k}=\left\{i \in \Gamma: n_{i}=k\right\}$ and $A_{k}=\bigoplus_{i \in \Gamma_{k}} T_{i}$. Then $T=$ $\bigoplus_{k=1}^{\infty} A_{k}$ and, for each $k \in \mathbb{N}, A_{k}$ is either absent or a regular nilpotent operator of order $k$. Then, by Proposition 2.7, $T \in(\mathrm{CS})$ if and only if $A_{k} \in(\mathrm{CS})$ for all $k \geq 1$.

Let $\varepsilon \in(0,1]$ be fixed. Note that $\left(A_{1}\right)_{\varepsilon}=A_{1}=0$ (if it exists) and by Lemma 5.1, the abnormal part of $\left(A_{k}\right)_{\varepsilon}$ is a regular nilpotent operator of order $k-1$ for $k \geq 2$. Then, by Proposition 2.7. $T_{\varepsilon} \in(\mathrm{CS})$ if and only if $\left(A_{k}\right)_{\varepsilon} \in(\mathrm{CS})$ for all $k \in \mathbb{N}$.

If $k \leq 3$, then, by Proposition [5.7 $\left(A_{k}\right)_{\varepsilon} \in$ (CS) for all $\varepsilon \in[0,1]$ if and only if $A_{k} \in(\mathrm{CS})$. Note that $A_{1}=0$ and, by Lemma 2.2, $A_{2} \in(\mathrm{CS})$. Since $\mathbf{a}^{t}=\mathbf{a}^{s}$ for all 2 -tuples a, by Theorem 3.3 , we deduce that $A_{3} \in(\mathrm{CS})$ if and only if $\operatorname{card}\left\{j \in \Gamma_{3}\right.$ : $\left.\mathbf{a}_{j}=\mathbf{a}\right\}=\operatorname{card}\left\{j \in \Gamma_{3}: \mathbf{a}_{j}=\mathbf{a}^{s}\right\}$ for all 2-tuples $\mathbf{a}$.

If $k \in \mathbb{N}$ and $k \geq 4$, then, by Proposition [5.10, $\left(A_{k}\right)_{\varepsilon} \in$ (CS) for all $\varepsilon \in[0,1]$ if and only if $\left|\lambda_{j}^{(i)}\right|=\left|\lambda_{j+2}^{(i)}\right|$ for all $i \in \Gamma_{k}$ and $1 \leq j \leq n_{i}-3=k-3$, and $\operatorname{card}\left\{i \in \Gamma_{k}\right.$ : $\left.\left(\left|\lambda_{1}^{(i)}\right|,\left|\lambda_{2}^{(i)}\right|, \cdots,\left|\lambda_{n_{i}-1}^{(i)}\right|\right)=\mathbf{a}\right\}=\operatorname{card}\left\{i \in \Gamma_{k}:\left(\left|\lambda_{1}^{(i)}\right|,\left|\lambda_{2}^{(i)}\right|, \cdots,\left|\lambda_{n_{i}-1}^{(i)}\right|\right)=\mathbf{a}^{s}\right\}$ for any $(k-1)$-tuple $\mathbf{a}$.

Similarly, one can prove the case " $T_{\varepsilon} \in(\mathrm{CS})$ for all $\varepsilon \in[0,1 / 2]$ ". This completes the proof.

Remark 5.12. By Proposition 3.5, Theorem 5.11 actually characterizes those unilateral weighted shifts $T$ satisfying $T_{\varepsilon} \in(\mathrm{CS})$ for all $\varepsilon \in[0,1]$.

Let $T \in \mathcal{B}(\mathcal{H})$ be a bilateral weighted shift with weighted sequence $\left\{w_{i}\right\}_{i \in \mathbb{Z}}$. If there exists some $k \in \mathbb{Z}$ such that $\left|w_{k-i}\right|=\left|w_{i}\right|$ for all $i \in \mathbb{Z}$, then we say that $T$ is complex symmetric with respect to $k$.

Theorem 5.13. Let $\left\{e_{i}\right\}_{i \in \mathbb{Z}}$ be an ONB of $\mathcal{H}$ and $T \in \mathcal{B}(\mathcal{H})$ with $T e_{i}=\lambda_{i} e_{i+1}$ for $i \in \mathbb{Z}$. If $\lambda_{i} \neq 0$ for all $i \in \mathbb{Z}$, then the following are equivalent:

(i) $T_{\varepsilon} \in(\mathrm{CS})$ for all $\varepsilon \in[0,1]$.

(ii) $T_{\varepsilon} \in(\mathrm{CS})$ for all $\varepsilon \in\left[0, \frac{1}{2}\right]$.

(iii) $\left|\lambda_{i}\right|=\left|\lambda_{i+2}\right|$ for all $i \in \mathbb{Z}$.

Proof. Without loss of generality, we may directly assume that $\left|\lambda_{i}\right|=\lambda_{i}$ for all $i \in \mathbb{Z}$.

"(i) $\Rightarrow$ (ii)". This is obvious.

"(iii) $\Rightarrow$ (i)". By hypothesis, we may assume that $\lambda_{2 i}=a$ and $\lambda_{2 i+1}=b$ for all $i \in \mathbb{Z}$. Arbitrarily choosing an $\varepsilon \in[0,1]$, a direct calculation shows that $T_{\varepsilon} e_{i}=$ $\mu_{i} e_{i+1}$, where $\mu_{i}=\lambda_{i}^{1-\varepsilon} \lambda_{i+1}^{\varepsilon}$ for all $i \in \mathbb{Z}$. Then $\mu_{2 i}=a^{1-\varepsilon} b^{\varepsilon}$ and $\mu_{2 i+1}=a^{\varepsilon} b^{1-\varepsilon}$ for all $i \in \mathbb{Z}$, and $T_{\varepsilon}$ is a bilateral weighted shift with weighted sequence $\left\{\mu_{i}\right\}_{i \in \mathbb{Z}}$. By Theorem 4.4 we have $T_{\varepsilon} \in(\mathrm{CS})$. Since $\varepsilon$ was arbitrarily chosen in $[0,1]$, we conclude the proof.

"(ii) $\Rightarrow$ (iii)". By Theorem 4.4, for each $\varepsilon \in\left[0, \frac{1}{2}\right]$, there exists $k_{\varepsilon} \in \mathbb{Z}$ such that $T_{\varepsilon}$ is complex symmetric with respect to $k_{\varepsilon}$. By an analysis of cardinality, one can see that there exist two distinct $\varepsilon_{1}, \varepsilon_{2} \in\left[0, \frac{1}{2}\right]$ such that $k_{\varepsilon_{1}}=k_{\varepsilon_{2}}$. Denote $k=k_{\varepsilon_{1}}=k_{\varepsilon_{2}}$. Note that $T_{\varepsilon_{j}} e_{i}=\left(\lambda_{i}^{1-\varepsilon_{j}} \lambda_{i+1}^{\varepsilon_{j}}\right) e_{i+1}$ for all $j=1,2$ and $i \in \mathbb{Z}$. Then we have $\lambda_{k-i}^{1-\varepsilon_{j}} \lambda_{k-i+1}^{\varepsilon_{j}}=\lambda_{i}^{1-\varepsilon_{j}} \lambda_{i+1}^{\varepsilon_{j}}$ for all $j=1,2$ and $i \in \mathbb{Z}$, that is,

$$
\left(\frac{\lambda_{k-i+1} \lambda_{i}}{\lambda_{i+1} \lambda_{k-i}}\right)^{\varepsilon_{j}}=\frac{\lambda_{i}}{\lambda_{k-i}}, \quad \forall j=1,2, i \in \mathbb{Z}
$$


Noting that $\varepsilon_{1} \neq \varepsilon_{2}$, then we deduce that $\lambda_{i}=\lambda_{k-i}$ and $\lambda_{k-i+1}=\lambda_{i+1}$ for all $i \in \mathbb{Z}$. A direct calculation shows that $\lambda_{i}=\lambda_{i+2}$ for all $i \in \mathbb{Z}$.

Lemma 5.14. Let $\left\{e_{i}\right\}_{i \in \mathbb{N}}$ be an ONB of $\mathcal{H}$. Assume that $A, B \in \mathcal{B}(\mathcal{H})$ and

$$
A e_{i}=\lambda_{i} e_{i+1}, \quad B e_{i}=\mu_{i} e_{i+1}
$$

for $i \in \mathbb{N}$. Set $T=A \oplus B^{*}$. If nul $A=0=\operatorname{nul} B$, then the following are equivalent:

(i) $T_{\varepsilon} \in(\mathrm{CS})$ for all $\varepsilon \in[0,1]$;

(ii) $T_{\varepsilon} \in$ (CS) for all $\varepsilon \in\left[0, \frac{1}{2}\right]$;

(iii) $T \in(\mathrm{CS})$ and there exists $\varepsilon \in(0,1 / 2)$ such that $T_{\varepsilon} \in(\mathrm{CS})$;

(iv) $\left|\lambda_{i+1}\right|=\left|\lambda_{i}\right|=\left|\mu_{i}\right|$ for all $i \in \mathbb{N}$.

Proof. Without loss of generality, we may assume that $\lambda_{i}>0$ and $\mu_{i}>0$ for all $i \in \mathbb{N}$.

The relations "(i) $\Rightarrow($ ii $) \Rightarrow($ iii)" are obvious. By Theorem 4.8, a simple calculation shows that the relation "(iv) $\Rightarrow$ (i)" holds. It suffices to prove that "(iii) $\Longrightarrow$ (iv)".

Assume that $\varepsilon \in(0,1 / 2)$ and $T_{\varepsilon} \in(\mathrm{CS})$. Note that

$$
A_{\varepsilon}=\sum_{i=1}^{\infty}\left(\lambda_{i}^{1-\varepsilon} \lambda_{i+1}^{\varepsilon}\right) e_{i+1} \otimes e_{i}, \quad\left(B^{*}\right)_{\varepsilon}=\sum_{i=2}^{\infty}\left(\mu_{i-1}^{\varepsilon} \mu_{i}^{1-\varepsilon}\right) e_{i} \otimes e_{i+1}
$$

Then, by Theorem 4.8. $T_{\varepsilon} \in$ (CS) implies $\lambda_{i}^{1-\varepsilon} \lambda_{i+1}^{\varepsilon}=\mu_{i}^{\varepsilon} \mu_{i+1}^{1-\varepsilon}$ for all $i \in \mathbb{N}$. On the other hand, by Theorem 4.8, $T \in(\mathrm{CS})$ implies that $\lambda_{i}=\mu_{i}$ for all $i \in \mathbb{N}$. Since $\varepsilon \in(0,1 / 2)$, we conclude that $\lambda_{i}=\lambda_{i+1}$ for all $i \in \mathbb{N}$. This completes the proof.

Theorem 5.15. Let $\left\{e_{i}\right\}_{i \in \mathbb{Z}}$ be an ONB of $\mathcal{H}$ and $T \in \mathcal{B}(\mathcal{H})$ with $T e_{i}=\alpha_{i} e_{i+1}$ for $i \in \mathbb{Z}$. If $0<\operatorname{card}\left\{i \in \mathbb{Z}: \alpha_{i}=0\right\}<\infty$, then the following are equivalent:

(i) $T_{\varepsilon} \in(\mathrm{CS})$ for all $\varepsilon \in[0,1]$.

(ii) $T_{\varepsilon} \in(\mathrm{CS})$ for all $\varepsilon \in\left[0, \frac{1}{2}\right]$.

(iii) $T \simeq(\lambda S) \oplus\left(\lambda S^{*}\right) \oplus A$, where $\lambda>0, S$ is the unilateral unweighted shift of multiplicity one, $A$ is absent, or $A$ is acting on a finite-dimensional Hilbert space with the following form:

$$
A=\left[\begin{array}{cccc}
0 & \lambda_{1} & & \\
& \ddots & \ddots & \\
& & 0 & \lambda_{n} \\
& & & 0
\end{array}\right]
$$

with respect to some ONB of the underlying space of $A$ and $A_{\varepsilon} \in(\mathrm{CS})$ for all $\varepsilon \in[0,1 / 2]$.

Proof. Without loss of generality, we may assume that $\alpha_{i} \geq 0$ for all $i \in \mathbb{Z}$. The relations "(i) $\Rightarrow$ (ii)" and "(iii) $\Rightarrow($ i)" are obvious. It suffices to prove that "(ii) $\Rightarrow$ (iii)".

By hypothesis, there exist $m, n \in \mathbb{Z}, m \leq n$, such that $\alpha_{m}=\alpha_{n}=0$ and $\alpha_{i}>0$ for all $i>n$ or $i<m$. We directly assume that $m<n$ (the case that $m=n$ is simpler). Denote $\mathcal{H}_{1}=\bigvee\left\{e_{i}: i \leq m\right\}, \mathcal{H}_{2}=\bigvee\left\{e_{i}: m<i \leq n\right\}$ and $\mathcal{H}_{3}=\bigvee\left\{e_{i}: i>n\right\}$. Then each $\mathcal{H}_{i}$ is a reducing subspace of $T$. For $1 \leq i \leq 3$, denote $T_{i}=\left.T\right|_{\mathcal{H}_{i}}$. Then $T_{1}^{*}$ and $T_{3}$ are two injective unilateral weighted shifts. Note that $T_{2}$ is nilpotent and $T_{2}^{k}=0$, where $k=n-m$.

For $\varepsilon \in[0,1]$, it can be seen from the proof of Theorem 4.8 that $T_{\varepsilon} \in(\mathrm{CS})$ if and only if $\left(T_{1}\right)_{\varepsilon} \oplus\left(T_{3}\right)_{\varepsilon} \in(\mathrm{CS})$ and $\left(T_{2}\right)_{\varepsilon} \in(\mathrm{CS})$. Then the conclusion follows immediately from Lemma 5.14 
Remark 5.16. By Theorem 4.9, if $A$ is a complex symmetric bilateral weighted shift with weighted sequence $\left\{\lambda_{i}\right\}_{i \in \mathbb{Z}}$ and $\operatorname{card}\left\{i \in \mathbb{Z}: \lambda_{i}=0\right\}=\infty$, then $A$ is also a unilateral weighted shift. Thus Theorem 5.11 combined with Theorems 5.13 and 5.15 completes the characterization of complex symmetric generalized Aluthge transforms of weighted shifts.

\section{REFERENCES}

[1] A. Aluthge, On p-hyponormal operators for $0<p<1$, Integral Equations Operator Theory 13 (1990), no. 3, 307-315. MR.1047771 (91a:47025)

[2] A. Aluthge, Some generalized theorems on p-hyponormal operators, Integral Equations Operator Theory 24 (1996), no. 4, 497-501. MR1382022 (97a:47032)

[3] L. Balayan and S. R. Garcia, Unitary equivalence to a complex symmetric matrix: geometric criteria, Oper. Matrices 4 (2010), no. 1, 53-76. MR2655004 (2011f:15068)

[4] J. A. Cima, S. R. Garcia, W. T. Ross, and Warren R. Wogen, Truncated Toeplitz operators: spatial isomorphism, unitary equivalence, and similarity, Indiana Univ. Math. J. 59 (2010), no. 2, 595-620. MR 2648079

[5] S. R. Garcia, Aluthge transforms of complex symmetric operators, Integral Equations Operator Theory 60 (2008), no. 3, 357-367. MR2392831 (2008m:47052)

[6] S. R. Garcia, Open questions about complex symmetric operators, Tech. report, Southeastern Analysis Meeting (SEAM 25), University of South Florida, Mar. 2009.

[7] S. R. Garcia and M. Putinar, Complex symmetric operators and applications, Trans. Amer. Math. Soc. 358 (2006), no. 3, 1285-1315 (electronic). MR2187654 (2006j:47036)

[8] S. R. Garcia and M. Putinar, Complex symmetric operators and applications. II, Trans. Amer. Math. Soc. 359 (2007), no. 8, 3913-3931 (electronic). MR2302518 (2008b:47005)

[9] S. R. Garcia and M. Putinar, Interpolation and complex symmetry, Tohoku Math. J. (2)60 (2008), no. 3, 423-440. MR2453732 (2009k:47048)

[10] S. R. Garcia and W. R. Wogen, Complex symmetric partial isometries, J. Funct. Anal. 257 (2009), no. 4, 1251-1260. MR2535469 (2011g:47005)

[11] S. R. Garcia and W. R. Wogen, Some new classes of complex symmetric operators, Trans. Amer. Math. Soc. 362 (2010), no. 11, 6065-6077. MR2661508 (2011g:47086)

[12] T. M. Gilbreath and W. R. Wogen, Remarks on the structure of complex symmetric operators, Integral Equations Operator Theory 59 (2007), no. 4, 585-590. MR2370050 (2009f:47037)

[13] J. Guyker, A structure theorem for operators with closed range, Bull. Austral. Math. Soc. 18 (1978), no. 2, 169-186. MR 499972 (80b:47020)

[14] P. R. Halmos and L. J. Wallen, Powers of partial isometries, J. Math. Mech. 19 (1969/1970), 657-663. MR0251574 (40:4801)

[15] D. A. Herrero, Approximation of Hilbert space operators. Vol. 1, second edition, Pitman Research Notes in Mathematics Series, vol. 224, Longman Scientific \& Technical, Harlow, 1989. MR1088255 (91k:47002)

[16] A. L. Shields, Weighted shift operators and analytic function theory, Topics in operator theory, Math. Surveys, No. 13, Amer. Math. Soc., Providence, R.I. 1974, pp. 49-128. MR 0361899 $(50: 14341)$

[17] X. H. Wang and Z. S. Gao, A note on Aluthge transforms of complex symmetric operators and applications, Integral Equations Operator Theory 65 (2009), no. 4, 573-580. MR.2576310 (2011c:47003)

[18] S. Zhu, C. G. Li, and Y. Q. Ji, The class of complex symmetric operators is not norm closed, Proc. Amer. Math. Soc. 140 (2012), no. 5, 1705-1708. MR.2869154

Department of Mathematics, Jilin University, Changchun 130012, People's Republic OF CHINA

Current address: School of Mathematical Sciences, Fudan University, Shanghai 200433, People's Republic of China

E-mail address: senzhu@163.com

Institute of Mathematics, Jilin University, Changchun 130012, People's Republic of CHINA

E-mail address: licg09@mails.jlu.edu.cn 